\title{
QUaD: A High-Resolution Cosmic Microwave Background Polarimeter
}

\section{Citation}

Hinderks, James R., Peter Ade, James Bock, Melanie Bowden, Michael L. Brown, Gary Cahill, John E. Carlstrom, and et al. 2009. QUaD: A high-resolution cosmic microwave background polarimeter. The Astrophysical Journal 692, no. 2: 1221-1246.

\section{Published Version}

doi:10.1088/0004-637X/692/2/1221

\section{Permanent link}

http://nrs.harvard.edu/urn-3:HUL.InstRepos:11129145

\section{Terms of Use}

This article was downloaded from Harvard University's DASH repository, and is made available under the terms and conditions applicable to Other Posted Material, as set forth at http:// nrs.harvard.edu/urn-3:HUL.InstRepos:dash.current.terms-of-use\#LAA

\section{Share Your Story}

The Harvard community has made this article openly available.

Please share how this access benefits you. Submit a story.

Accessibility 


\title{
QUaD: A HIGH-RESOLUTION COSMIC MICROWAVE BACKGROUND POLARIMETER
}

\author{
J. R. Hinderks ${ }^{1,10}$, P. Ade ${ }^{2}$, J. Bock ${ }^{3,4}$, M. Bowden ${ }^{1}$, M. L. Brown ${ }^{5,11}$, G. Cahill ${ }^{6}$, J. E. Carlstrom ${ }^{7}$, P. G. Castro ${ }^{5,12}$, \\ S. $\mathrm{Church}^{1}$, T. Culverhouse ${ }^{7}$, R. Friedman ${ }^{7}$, K. GanGa ${ }^{8}$, W. K. Gear ${ }^{2}$, S. Gupta ${ }^{2}$, J. Harris ${ }^{2}$, V. Haynes ${ }^{2}, 13$, B.

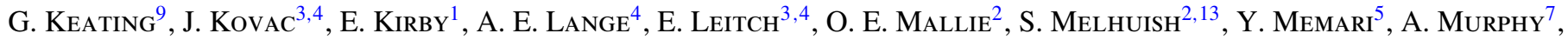 \\ A. Orlando ${ }^{2,4}$, R. Schwarz ${ }^{7}$, C. O' Sullivan $^{6}$, L. Piccirillo ${ }^{2,13}$, C. Pryke ${ }^{7}$, N. Rajguru ${ }^{14}$, B. Rusholme ${ }^{1,3}$, A. N. TaYlor ${ }^{5}$, \\ K. L. Thompson ${ }^{1}$, C. TuCKer ${ }^{2}$, A. H. Turner ${ }^{2}$, E. Y. S. Wu ${ }^{1}$, And M. ZemCov ${ }^{3,4}$ \\ ${ }^{1}$ Kavli Institute for Particle Astrophysics and Cosmology and Department of Physics, Stanford University, 382 Via Pueblo Mall, Stanford, CA 94305, USA \\ ${ }^{2}$ School of Physics and Astronomy, Cardiff University, Queen's Buildings, The Parade, Cardiff CF24 3AA, UK \\ 3 Jet Propulsion Laboratory, 4800 Oak Grove Dr., Pasadena, CA 91109, USA \\ ${ }^{4}$ California Institute of Technology, Pasadena, CA 91125, USA \\ ${ }^{5}$ Institute for Astronomy, University of Edinburgh, Royal Observatory, Blackford Hill, Edinburgh EH9 3HJ, UK \\ ${ }^{6}$ Experimental Physics, National University of Ireland, Maynooth, Ireland

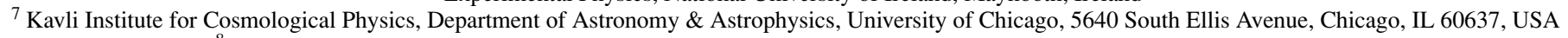 \\ ${ }^{8}$ Laboratoire APC/CNRS; Bâtiment Condorcet; 10, rue Alice Domon et Léonie Duquet, 75205 Paris Cedex 13, France \\ ${ }^{9}$ Center for Astrophysics and Space Sciences, University of California, San Diego, 9500 Gilman Drive, La Jolla, CA 92093, USA \\ Received 2008 May 14; accepted 2008 October 2; published 2009 February 24
}

\begin{abstract}
We describe the QUaD experiment, a millimeter-wavelength polarimeter designed to observe the cosmic microwave background (CMB) from a site at the South Pole. The experiment comprises a $2.64 \mathrm{~m}$ Cassegrain telescope equipped with a cryogenically cooled receiver containing an array of 62 polarization-sensitive bolometers. The focal plane contains pixels at two different frequency bands, $100 \mathrm{GHz}$ and $150 \mathrm{GHz}$, with angular resolutions of $5^{\prime}$ and 3'5, respectively. The high angular resolution allows observation of CMB temperature and polarization anisotropies over a wide range of scales. The instrument commenced operation in early 2005 and collected science data during three successive Austral winter seasons of observation.
\end{abstract}

Key words: cosmic microwave background - instrumentation: polarimeters

Online-only material: color figures

\section{INTRODUCTION}

The cosmic microwave background (CMB) remains a key tool for understanding the origin and evolution of the universe. Thompson scattering from quadrupole anisotropies at the surface of last scattering polarizes the CMB at the level of $10 \%$. The resulting polarization pattern on the sky can be mathematically decomposed into even-parity $\mathrm{E}$ modes and odd-parity B modes (Zaldarriaga \& Seljak 1997). The E-mode signal, which has been detected by a number of experiments (Readhead et al. 2004; Leitch et al. 2005; Montroy et al. 2006; Page et al. 2007; Wu et al. 2007; CAPMAP Collaboration: C. Bischoff et al. 2008), is dominated by scalar perturbations (density fluctuations) in the early universe. A B-mode signal has yet to be detected but could be generated by gravitational waves in the early universe or lensing of $\mathrm{E}$ modes by intervening structure.

This paper describes $\mathrm{QUaD},{ }^{15}$ a polarimeter designed to observe the CMB. QUaD comprises a bolometric receiver located on a $2.64 \mathrm{~m}$ telescope near the geographic South

\footnotetext{
${ }^{10}$ Current address: NASA Goddard Space Flight Center, 8800 Greenbelt Road, Greenbelt, Maryland 20771, USA

${ }^{11}$ Current address: Cavendish Laboratory, University of Cambridge, J.J. Thomson Avenue, Cambridge CB3 OHE, UK.

${ }^{12}$ Current address: CENTRA, Departamento de Física, Edifício Ciência, Instituto Superior Técnico, Universidade Tecnica de Lisboa, Av. Rovisco Pais 1, 1049-001 Lisboa, Portugal.

${ }^{13}$ Current address: School of Physics and Astronomy, University of Manchester, Manchester M13 9PL, UK.

${ }^{14}$ Current address: Department of Physics and Astronomy, University College London, Gower Street, London WC1E 6BT, UK.

15 QUaD stands for QUEST (QU Extragalactic Survey Telescope) at DASI (Degree Angular Scale Interferometer).
}

Pole. The QUaD receiver contains a focal plane array of 31 pixels, each composed of a corrugated feed horn and a pair of orthogonal polarization-sensitive bolometers (PSBs). Each pixel simultaneously measures both temperature and one linear polarization Stokes parameter. The pixels are divided between two observing frequencies with 12 at $100 \mathrm{GHz}$ and 19 at $150 \mathrm{GHz}$. The angular resolution is $5^{\prime} .0$ at $100 \mathrm{GHz}$ and 3.5 at $150 \mathrm{GHz}$, and the instantaneous field of view is 1.5 . Table 1 gives the optical properties of the telescope and receiver. First light was in 2005 February, and three seasons of Austral winter observations were completed before the instrument was decommissioned in late 2007. Results from the first season's data are presented in Ade et al. (2008). Results from the second and third seasons' data are presented in Pryke et al. (2008), a companion paper that will be referred to in this work as the Data Paper.

This paper is organized as follows: Sections 2 and 3 review the observing site, telescope mount, and optics. Sections 4-6 describe the focal plane, cryogenics, and readout electronics. Section 7 presents the performance of the instrument as measured in the laboratory and the field. Section 8 describes the measures taken to mitigate interfering signals. Section 9 describes the calibration procedures for the instrument. Section 10 discusses the instrument sensitivity.

\section{OBSERVING SITE AND TELESCOPE MOUNT}

QUaD is sited at the Martin A. Pomerantz Observatory (MAPO), part of the Amundsen-Scott Station, $0.7 \mathrm{~km}$ from the geographic South Pole. The Observatory is atop the Antarctic Plateau at a physical elevation of $2800 \mathrm{~m}$ and at an equivalent 


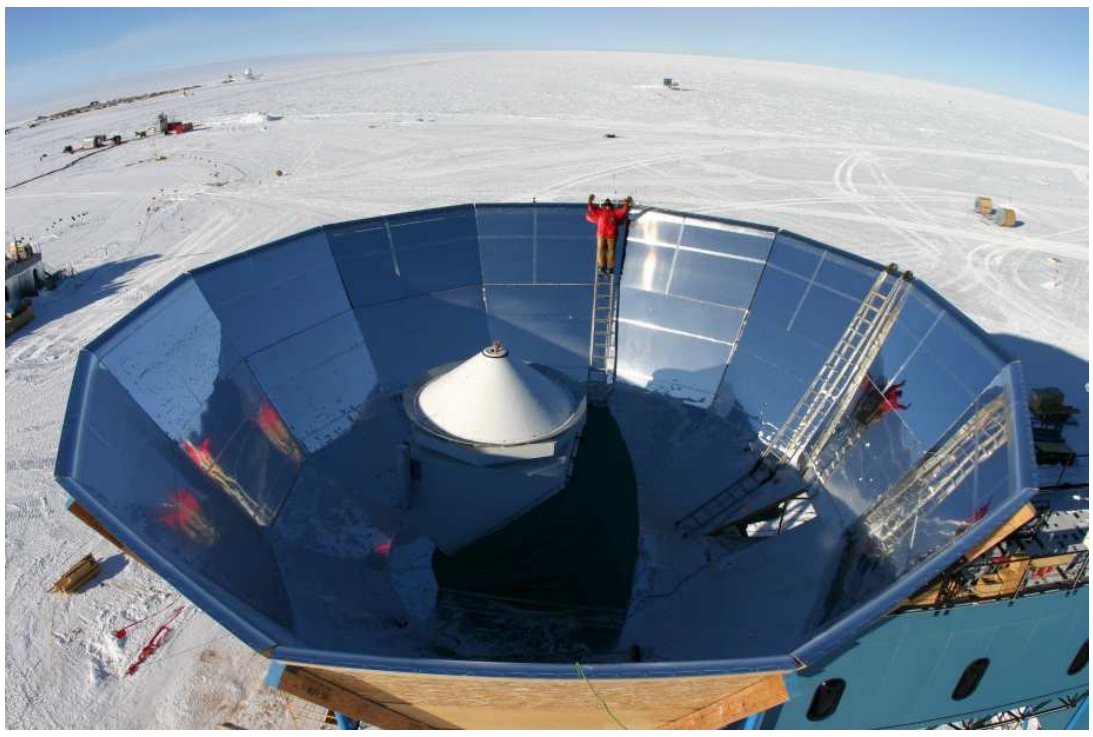

Figure 1. QUaD telescope within the reflective ground shield.

(A color version of this figure is available in the online journal.)

Table 1

Optical Properties of the QUaD Telescope and Receiver

\begin{tabular}{lccc}
\hline \hline Telescope & & & \\
Primary mirror diameter (m) & & 2.64 & \\
Secondary mirror diameter (m) & & 0.45 & \\
Total field of view (deg) & & 0.5 & \\
Pointing accuracy (arcmin) & 100 & & 150 \\
Nominal frequency bands (GHz) & 5.0 & & 3.5 \\
Beam FWHM (arcmin) & & & \\
Receiver & 94.6 & & 149.5 \\
Band center (GHz) & 26 & & 41 \\
Bandwidth (GHz) & 24 & & 38 \\
Number of detectors & 12 & & 19 \\
Number of pixels & 9 & & 18 \\
Operating pixels $(06,07)$ & 27 & & 34 \\
Optical efficiency (\%) & 30 & \\
Time constant (ms) & 8 & \\
Cross-polar leakage (\%) & & 80 \\
\end{tabular}

Note. Parameters listed here are average values for the band. The details are provided in Section 7.

pressure elevation in excess of $3200 \mathrm{~m}$. This is recognized as a premier site for ground-based millimeter-wave observations (Lane 1998; Lay \& Halverson 2000; Peterson et al. 2003; Bussmann et al. 2005). The low temperature freezes out much of the remaining precipitable water vapor above the telescope, reducing emission and absorption at millimeter wavelengths. The temperature and atmosphere are stable over long periods of time with minimal diurnal variation during the six-month darkness of the Austral winter. The site also affords unchanging access to the Southern Celestial Hemisphere, allowing deep and continuous integration on the target region with rigorous control of ground-spill systematics.

Figure 1 shows the observatory and Figure 2 shows a schematic of the experiment. The telescope is located on the mount previously used by the DASI experiment (Leitch et al. 2002a, 2002b). The mount is an altitude-azimuth design, with an additional axis of rotation that allows the entire telescope and receiver to be rotated about the optical axis of the instrument. Known as "deck" rotation, which allows each detector to

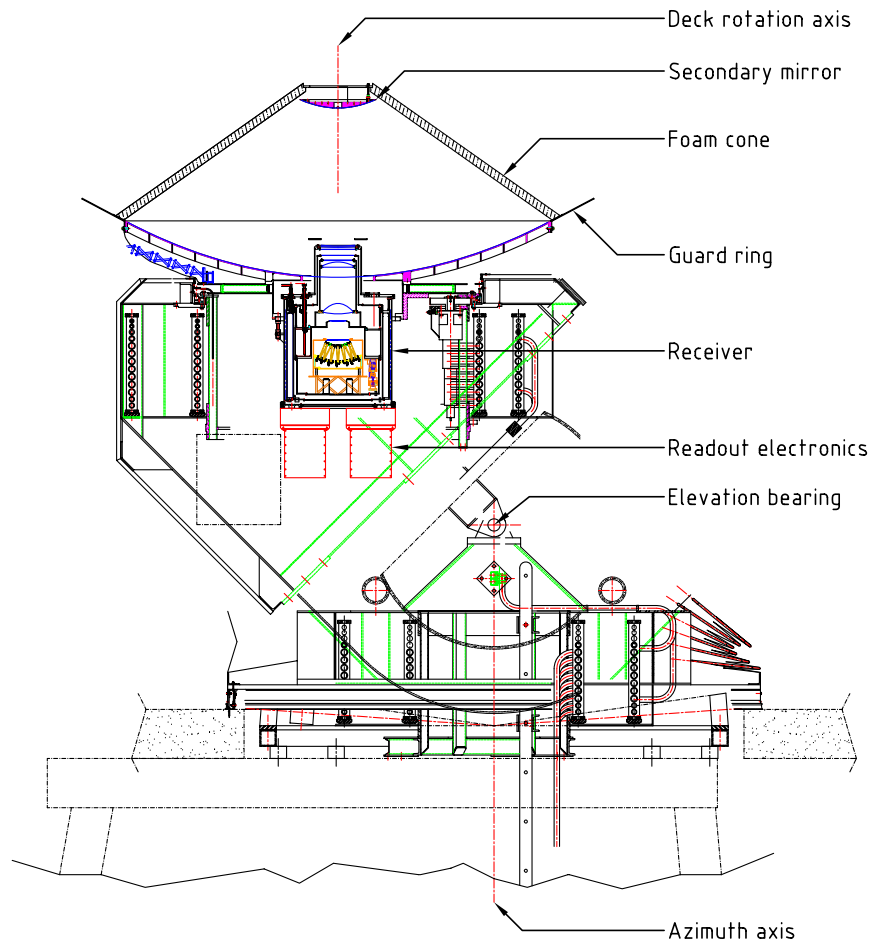

Figure 2. Schematic of the telescope and receiver.

(A color version of this figure is available in the online journal.)

view the sky at multiple polarization angles, despite the fixed parallactic angle of sources viewed from the Polar location. Observations are made by scanning the telescope in azimuth, stepping in elevation between scans.

The telescope mount is isolated from wind loading and other sources of vibration by being situated on the inner of two concentric, mechanically isolated towers. The outer tower supports the reflective ground shield and is connected to the observatory building. The receiver, readout electronics, and cryogen lines for filling are accessed from a heated room below the mount. This arrangement minimizes outdoor activity, which is difficult in winter due to darkness and extreme cold (as low as $-80^{\circ} \mathrm{C}$ ambient). 


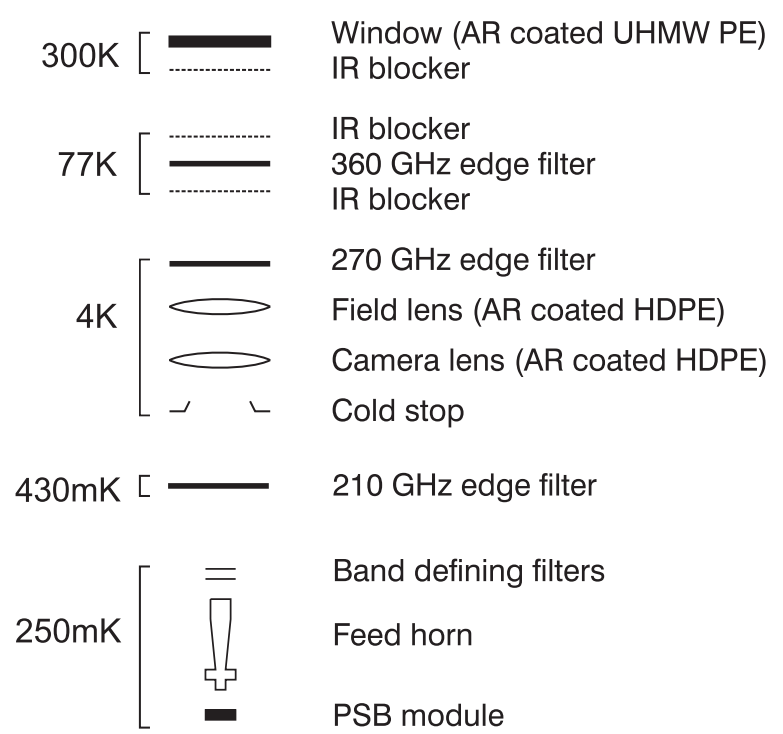

Figure 3. Receiver optics and filter chain. The window is made from AR-coated UHMW PE. The lenses are AR-coated HDPE.

\section{OPTICS}

The QUaD telescope is an axisymmetric Cassegrain design. The warm fore optics comprise a parabolic primary mirror and hyperbolic secondary. The upward-looking cryogenic receiver contains two cooled reimaging lenses, a cold stop at the image of the primary, and a curved focal plane. Figure 3 details the receiver optics chain. The design requirements were for high image quality (Strehl ratios $>0.98$ ) over a large (1.5) field of view, and a cold stop for sidelobe control. Minimizing the secondary blockage required curving the focal plane and moving the field lens above the primary.

The optical design was primarily performed with the ZEMAX ray-tracing software. Gaussian beam mode analysis and the GRASP8 physical optics package were used for final optimization, and to investigate the effects of diffraction. The optical design process is described in greater detail in Cahill et al. (2004) and O'Sullivan et al. (2008).

\subsection{Telescope Optics}

The telescope design, including the use of a foam cone to support the secondary mirror, is closely based on the COMPASS experiment (Farese et al. 2004). The $2.64 \mathrm{~m}, \mathrm{~F} / 0.5$ primary mirror ${ }^{16}$ is molded from a single plate of aluminum with support ribs welded on the rear surface and is identical to the one used in COMPASS. To minimize movement of the foam cone when the telescope elevation is changed, the secondary mirror assembly was made as light as possible. For this reason, the $0.45 \mathrm{~m}$ secondary mirror was manufactured from a thin sheet of aluminum supported by a carbon fiber backing. ${ }^{17}$

Spillover and sidelobes are a concern for any on-axis telescope design. Several steps, outlined here, were taken to mitigate their effects. The receiver optics (see Section 4.2) illuminate the primary with a Gaussian pattern and a $-20 \mathrm{~dB}$ edge taper. A thin aluminum guard ring was used to extend the primary radius by $0.3 \mathrm{~m}$ to reflect spillover onto cold sky. The secondary mirror has a $48 \mathrm{~mm}$ hole in its center to prevent reillumination of the receiver window by the central portion of the beams from the

\footnotetext{
$\overline{16}$ Costruzioni Ottico-Meccaniche MARCON, Italy

17 Forestal SRL, 248 Via Di Salone, Rome, Italy
}

receiver. The hole is also used to periodically inject a calibration signal into the data stream from a source located behind the secondary mirror. Additionally, an annular aluminum reflecting collar surrounds the window of the cryostat, filling the geometric shadow of the secondary. Finally, the telescope is located within a reflective ground shield that blocks line-of-sight contact between the primary mirror and the ground. To prevent excess loading from snow accumulation, the ground shield and foam cone were inspected and swept daily, and detailed logs of snow buildup were kept. Section 8.5 describes measurements of the sidelobes and their effects.

\subsection{The Foam Cone}

The primary reason for using a foam cone to support the secondary mirror was to preserve the axial symmetry of the instrument which would have been broken by discrete supporting legs. However, the cone conferred an extra advantage by trapping warm air venting up from the heated room below in the space between the cone and the mirror. This warm air kept the components inside the cone (the receiver snout, secondary assembly, and calibration source) at $\sim 15{ }^{\circ} \mathrm{C}$, even during the extreme cold of the polar winter. This protected both the primary and secondary mirrors, and the cryostat window, from icing and contraction issues and removed concerns about the cryostat O-rings freezing.

After testing for millimeter-wave transmission, mechanical stiffness, and weather resistance, Zotefoam PPA30 (a closed cell propazote foam expanded with dry nitrogen gas) was selected as the most suitable material for the cone. The cone was constructed from two layers of 1". 1 Zotefoam bonded with Scotch 924 film transfer adhesive. Each layer is composed of nine sections that were cut from flat sheets and molded into the appropriate shape by pressing them between machined aluminum forms while baking them in a custom oven. The cone assembly was performed on a full-size wooden mandrel. The two layers of foam are rotated so that the sector seams are antialigned. After assembly, the cone and mandrel were placed in a large plastic bag which was then evacuated to apply uniform pressure simultaneously over the full cone surface, producing excellent adhesion. A fiberglass mounting collar clamps the cone along the bottom edge and provides an attachment point where it is bolted to the primary mirror guard ring. A similar clamping collar along the top edge forms a mount for the secondary mirror assembly.

As manufactured, Zotefoam sheets have a $\sim 00^{\prime} .1$ thick overdense skin on both surfaces. For improved transmission, the QUaD cone was assembled from sheets that had the skin removed. Reflection off a sample section of the cone (including two Zotefoam layers and adhesive) was measured to be $2 \%$ at $150 \mathrm{GHz}$. This reflection coefficient, though small, creates a narrow annular sidelobe at $100^{\circ}$ from the telescope boresight (Section 8.5).

\subsection{Cryogenic Optics}

The cryogenic optics couple the Cassegrain focus of the telescope onto the detectors and form an image of the primary mirror at the cold stop. Figure 4 shows the optics mounted in the receiver. The two lenses are $18 \mathrm{~cm}$ in diameter and are made from high-density polyethylene $\left(\mathrm{HDPE}^{18}\right)$. The relatively low refractive index of HDPE is desirable to limit losses due to surface reflection. The room temperature index of a

\footnotetext{
18 Professional Plastics, www.professionalplastics.com
} 


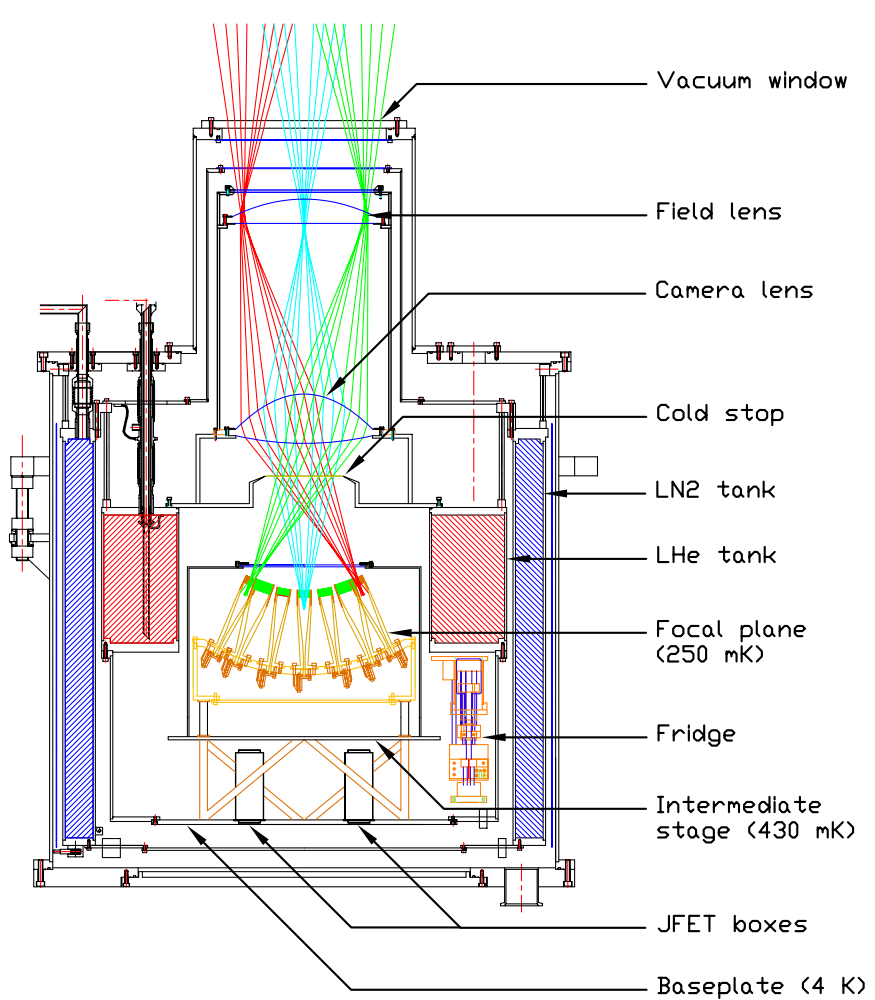

Figure 4. Schematic of the cryogenic receiver. Rays are shown for the central and two outer pixels.

(A color version of this figure is available in the online journal.)

sample of the HDPE used for the QUaD lenses was measured using a Fourier Transform Spectrometer (FTS) to be $n=$ $1.5413 \pm 0.0007$ at $150 \mathrm{GHz}$. Using published data on the temperature dependence of the refractive index of HDPE (Birch $\&$ Ping 1984) this was scaled to 1.583 at $4 \mathrm{~K}$. A consequence of the low refractive index of HDPE is that the lenses are highly curved.

The lenses are antireflection (AR)-coated with a thin film of porous PTFE, resulting in negligible loss, and are cooled to minimize loading on the detectors. The location of the field lens complicates cooling, requiring a snout on the cryostat that protrudes through the hole in the primary mirror. The lenses are mounted coaxially in an OFHC copper tube that is thermally anchored to the top of the Helium tank. The temperature of the top lens is $6.2 \mathrm{~K}$ after a cryogen fill and increases by less than $1 \mathrm{~K}$ over the course of an $18 \mathrm{hr}$ observing run as the liquid helium level decreases.

Corrugated feeds couple the optical signal from the lenses onto the polarization-sensitive bolometric detectors. These focal-plane optical components are described in detail in the next section. A $4 \mathrm{~K}$ knife-edged cold stop, located at a pupil between the camera lens and the focal plane, truncates the feed horn beams at approximately $-20 \mathrm{~dB}$ to prevent the sidelobes from viewing warm elements further down the optical chain. The underside of the cold stop is coated in a thin layer of carbonloaded Stycast for increased absorption (Bock 1994).

\section{THE FOCAL PLANE}

The QUaD focal plane (Figure 5) is a dual-frequency array of 31 pixels, each composed of a corrugated feed horn and a pair of orthogonally oriented, PSBs. The focal plane assembly is cooled to $\sim 250 \mathrm{mK}$ with a three-stage sorption fridge and

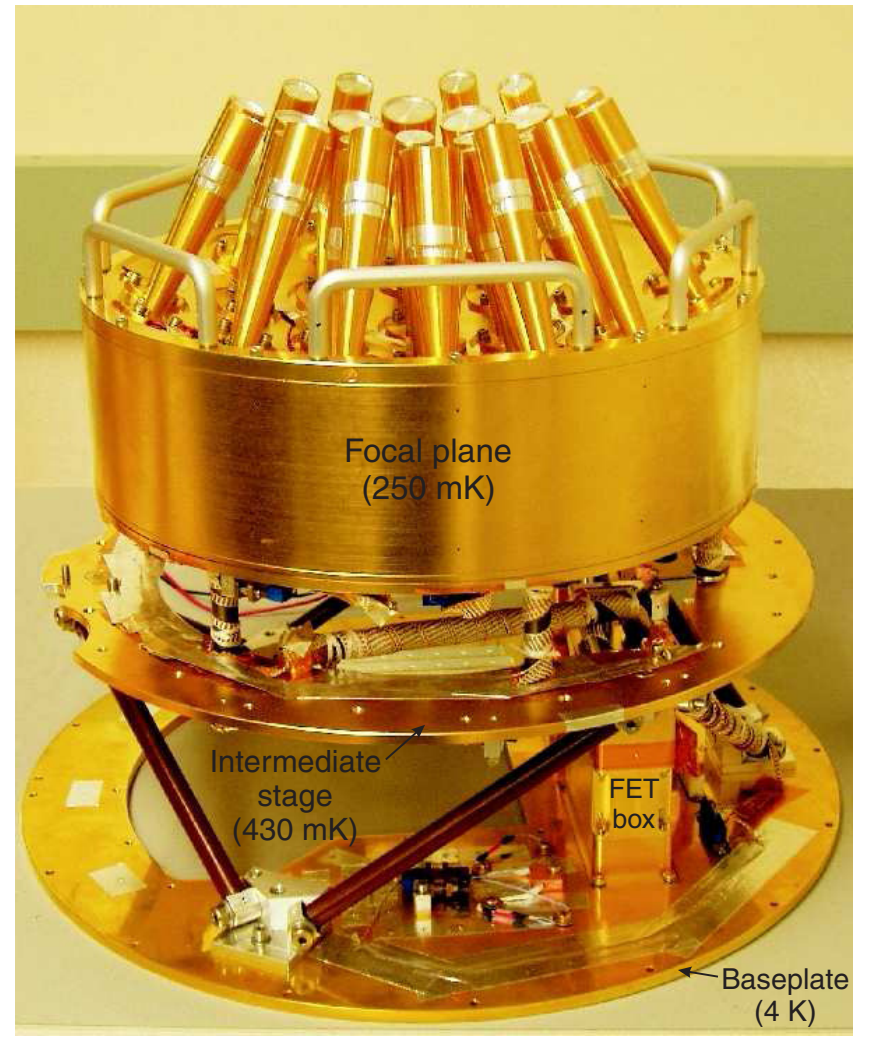

Figure 5. QUaD receiver core including the focal plane, JFET boxes, hexapod, and cold wiring. The feed horns and cylindrical light-tight bolometer enclosure are at $250 \mathrm{mK}$. Six Vespel legs separate this stage from the intermediate temperature $(430 \mathrm{mK})$ stage below. A Vespel hexapod isolates the intermediate stage from the $4 \mathrm{~K}$ baseplate. Low thermal conductivity ribbon cable, wrapped around the Vespel legs, connects the focal plane to the JFET boxes and reads out thermometers located on both sub-Kelvin temperature stages. The cutout in the $4 \mathrm{~K}$ baseplate is for access to attach the focal plane to the fridge during installation. Note, in this photograph, optical filters had yet to be installed on the feed horns, which are shown blanked off with Eccosorb-backed aluminum disks for dark testing.

(A color version of this figure is available in the online journal.)

is supported from the $4 \mathrm{~K}$ baseplate by a two-tiered structure made from low thermal conductivity plastic (Section 5).

The feed horns are arranged in a hexagonal configuration (Figure 6) and are positioned so that their phase centers lie along the spherical focal surface created by the optics. Nineteen of the feeds operate at $150 \mathrm{GHz}$ and the remaining 12 operate at $100 \mathrm{GHz}$. The focal plane bowl (radius of $175 \mathrm{~mm}$ ), which supports the feeds, was milled from a single piece of aluminum 6061 and gold plated to improve thermal conductivity.

Each feed terminates in a pair of orthogonal PSBs that detect the incident radiation. Summing the two PSB voltages from a pair results in a signal proportional to the total intensity. Subtracting the two voltages produces a measurement that is a linear combination of Stokes $Q$ and $U$, where the relative proportion depends on the orientation angle of the PSB with respect to the sky (Equation (5)). In order to determine the values of both $Q$ and $U$ for a given spot on the sky, it must be observed at two different orientation angles. For QUaD, the voltage from each PSB is independently recorded. Summing and differencing is performed in software during post-processing.

Maps of the sky are made by scanning the telescope in azimuth with the detector rows aligned along the scan direction. The pixel orientations were chosen so that a given location on the sky is observed at two different angles by the detectors in a 


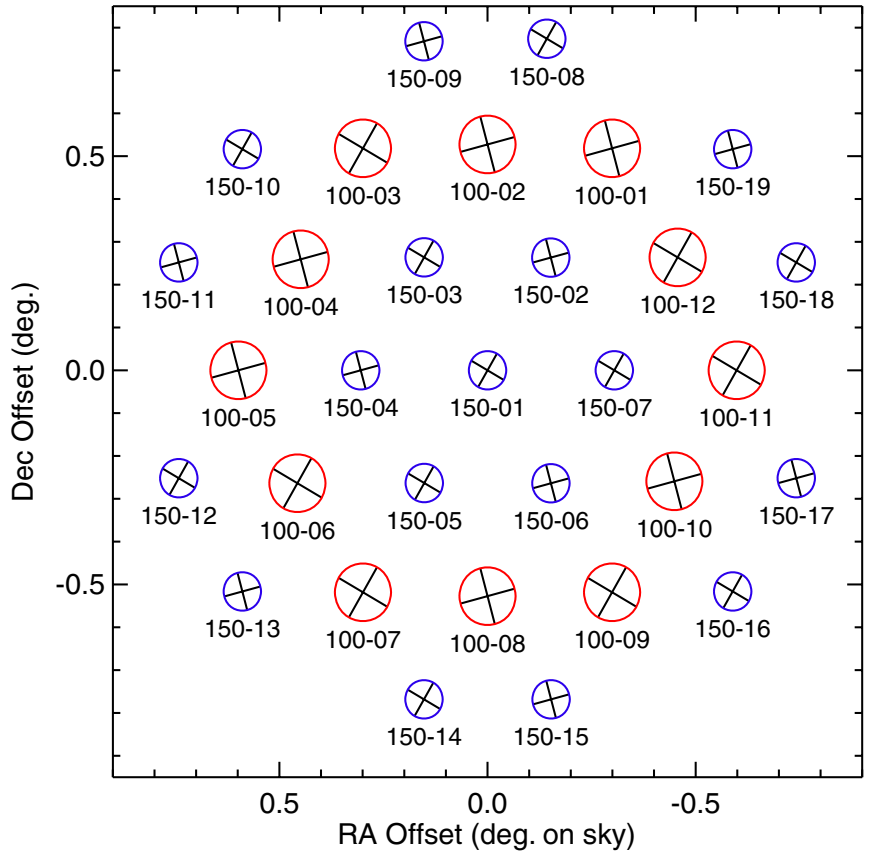

Figure 6. Layout of the QUaD PSBs, showing the relative direction of polarization to which each pixel is sensitive. The $150 \mathrm{GHz}$ pixels are in blue, the $100 \mathrm{GHz}$ in red.

(A color version of this figure is available in the online journal.)

given row during each scan. Additionally, two different values of the telescope "deck" rotation angle $\left(0^{\circ}\right.$ and $\left.60^{\circ}\right)$ were used while mapping to better constrain the polarization angles of the source and as a check for systematic effects.

A cylindrical light shield, also made from aluminum, surrounds the underside of the focal plane bowl and encloses the detectors, load resistor boards (Section 6.1), and miscellaneous thermometry. Two thermistors and three heater resistors are mounted on the focal plane bowl and are used with an external control system to stabilize the focal plane temperature during operation (Section 5.4). Four "dark" PSB modules are mounted to the back of the focal plane and are used to monitor for nonoptically induced contamination due to, for example, temperature drifts or electrical pickup. Several fixed resistors are also included and are used to monitor the noise in the readout electronics.

\subsection{Spectral Bands and Filtering}

The two QUaD bands of 78-106 and $126-170 \mathrm{GHz}$ were chosen to span atmospheric windows of high transparency (Figure 7). They are near the maximum of the $2.7 \mathrm{~K} \mathrm{CMB}$ blackbody spectrum and the minimum of polarized foreground contamination from galactic dust and synchrotron emission (Kogut et al. 2007). Observing at two frequencies allows the level of foreground contamination in the final maps to be investigated.

The band edges are set entirely using optical methods. The waveguide cutoff in the narrow throat section of each feed horn sets the lower band edge (Section 4.2) while metal-mesh lowpass filters on the horn apertures set the upper band edge. The low-pass filters were manufactured at Cardiff University using photolithography to pattern capacitative structures on thin layers of vacuum-deposited metal over a polypropylene substrate (Ade et al. 2006). Multiple layers with staggered cutoff frequencies are required to block leaks that occur at the harmonics and give

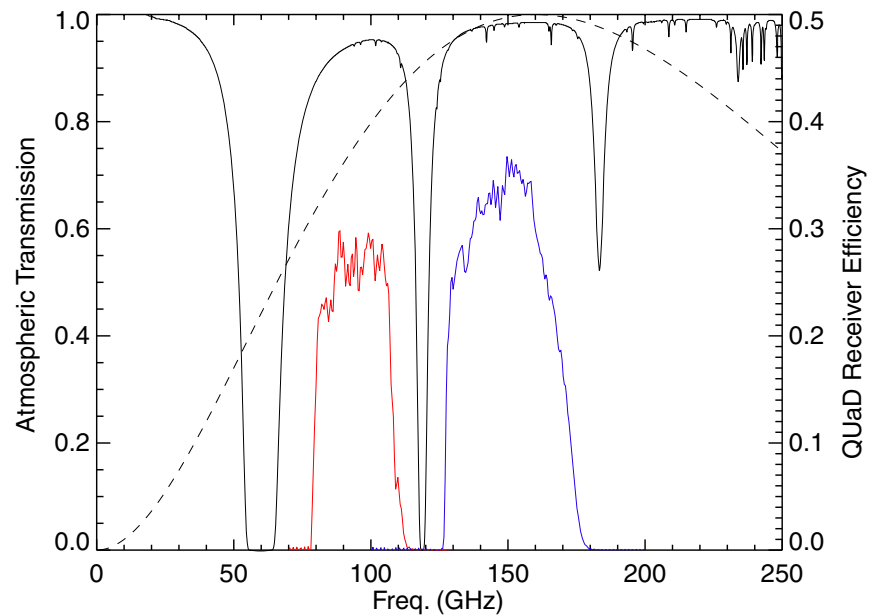

Figure 7. QUaD average spectral bands (red, blue), the South Pole atmospheric transmission (solid black), and the CMB spectrum (dashed black). The QUaD bands are normalized in terms of absolute transmission per polarization referring to the scale on the right.

(A color version of this figure is available in the online journal.)

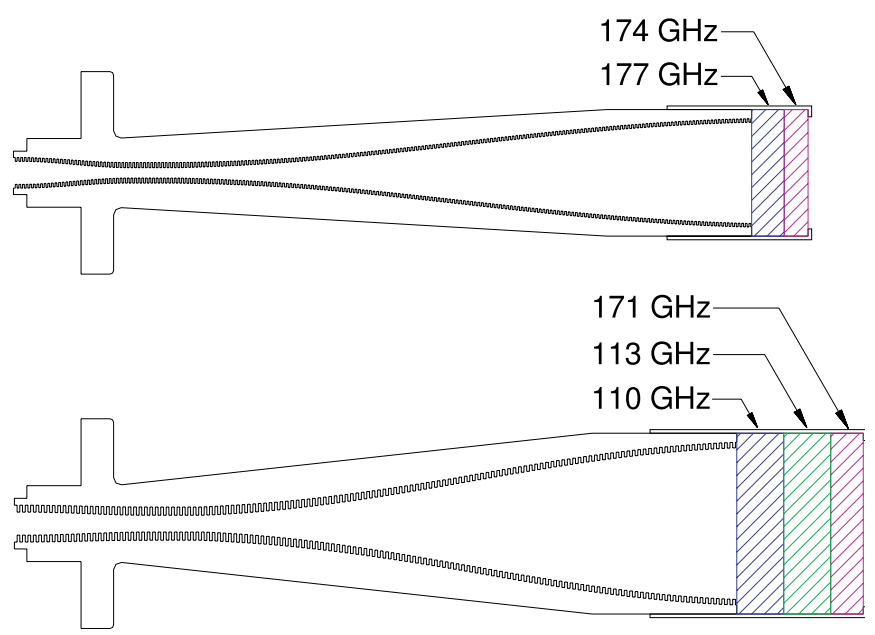

Figure 8. Schematic of the QUaD corrugated feeds, band-defining filters, and filter caps. The length of the $100 \mathrm{GHz}(150 \mathrm{GHz})$ feed is $100 \mathrm{~mm}(102 \mathrm{~mm})$, not including the filters and filter cap.

(A color version of this figure is available in the online journal.)

the required rejection of out-of-band power. The filters are AR coated to improve in-band transmission.

The particular filter combinations at each frequency (Figure 8) were chosen after extensive testing of different filters in a single-pixel optical test bed. An FTS was used to measure the band shape and thick grill filters were used to check for out-of-band leaks. ${ }^{19}$ FTS measurements of all feeds were made prior to shipping the receiver to the Pole and again before the receiver was installed on the telescope. By adding or removing filters from the chain during laboratory testing we were able to verify that the filters contribute negligible cross-polar leakage (Section 7.6).

Blocking filters that reflect high-frequency out-of-band radiation are located on each thermal stage within the cryostat, reducing the radiative load on the colder stages. They are held in place with aluminum clamping rings that are screwed down

\footnotetext{
19 Thick-grill filters are metal plates with drilled holes that act as high-pass filters via their waveguide cutoff. Filters with holes sized to cut on just above the band of interest allows the above-band power to be measured.
} 
for good thermal contact. The filters at the horn apertures are secured with a thin-walled brass filter cap that slides over the horn body and is itself held in place with the aluminum tape. A beryllium copper wavy spring washer provides thermal contact between the filter stack and the horn mouth, and a thin ring of indium wire seals the filters against the cap to prevent high-frequency optical leaks.

\subsection{Feed Horns}

Corrugated feed horns couple the telescope optics to the detectors. The $\lambda / 4$ corrugations allow the hybrid $\mathrm{HE}_{11}$ mode, which is a linear combination of the $\mathrm{TE}_{11}$ and $\mathrm{TM}_{11}$ circular guide modes, to propagate. This mode possesses a nearly axially symmetric field amplitude that smoothly tapers to zero along the horn walls, resulting in nearly symmetric $\mathrm{E}$ and $\mathrm{H}$ plane beam patterns, low cross-polar response, and low sidelobes. Consequently this type of horn is ideal for millimeter-wave polarimetry.

Thomas Keating Ltd. $^{20}$ manufactured the horns by electroforming copper onto CNC-machined aluminum mandrels, which were subsequently dissolved. Figure 8 shows a crosssectional schematic of the QUaD horns. The feeds were designed to be roughly matched in length at each frequency to prevent shadowing effects on the focal plane. Note that QUaD employs "profiled" feeds in which the diameter expands nonlinearly along the length of the horn. Profiling allows the horns to be shorter than a conical feed with equivalent sidelobe levels (Gleeson et al. 2005). This is particularly important for QUaD because the curved focal surface causes both the height and diameter of the required cold volume in the receiver to depend on the feed length.

The horn beams were modeled using a waveguide mode matching technique and the resulting predictions were checked against measurements of prototype horns (Figure 9). The horns were designed to illuminate the cold stop with an edge taper of approximately $-20 \mathrm{~dB}$; however when the filter cap, the filters, and the effects of the broad bandwidth are included, the feed beams are slightly wider. See Murphy et al. (2005) for further detail on the design, numerical modeling, and experimental verification of the QUaD corrugated feeds.

The narrow waveguide throat acts as a high-pass filter, defining the lower edge of the frequency band (Section 4.1). Gleeson et al. (2005) provides more detail on design considerations for this section of the feed. After the throat, the back end of the feed flares outward to couple the field onto the PSB absorber (Section 4.3). Modeling of the feed indicates a peak cross-polar level $\sim 30 \mathrm{~dB}$ down from the copolar beam, well below the levels introduced by the detectors.

\subsection{Polarization-Sensitive Bolometers}

Bolometers detect incident optical power by measuring the temperature increase of an absorber that is weakly thermally connected to a fixed bath temperature. QUaD uses PSBs where the absorber is patterned as an array of parallel line conductors, so that it only couples to one linear polarization (Figure 10). These detectors were fabricated at the JPL Micro Devices Laboratory, where similar detectors were developed for the Planck satellite (Jones et al. 2003) and the BOOMERANG experiment (Masi et al. 2006). PSBs identical to those in QUaD are used by the BICEP experiment (Yoon et al. 2006).

\footnotetext{
20 Station Mills, Billingshurst, West Sussex, RH14 9SH, UK
}

The PSB membranes are fabricated from silicon-nitride $\left(\mathrm{Si}_{3} \mathrm{~N}_{4}\right)$ and metalized with $120 \AA$ of gold over $20 \AA$ of titanium. The width of the conductors and their metalization thickness were optimized for maximum coupling using numerical simulation and experimentation with trial devices at Caltech/ JPL. The conductor spacing of $150 \mu \mathrm{m}$ allows the same membranes to be used at both observing frequencies. Nonmetalized legs run perpendicular to the conductors for mechanical support and have negligible impact on the optical properties (Jones et al. 2003).

An array of nonmetalized radial spokes support the circular absorber. A neutron-transmutation-doped (NTD) Ge thermistor $^{21}$ monitors its temperature. The thermistor is located on a conducting ring that runs along the circumference of the absorber. The ring lies outside the optical coupling area and provides a thermal connection between the parallel conducting traces of the absorber.

The dominant thermal path to the absorber is through three thicker legs near the thermistor, the central of which also carries the electric signals. These legs were designed to be laser ablated, if necessary, allowing the overall thermal conductance between the absorber and the focal plane, known as $G$, to be tuned. This operation was not performed for QUaD.

For ease of handling and attachment to the feed horns, pairs of orthogonally oriented PSBs are mounted in protective brass modules. The module forms a cylindrical integrating cavity, $\lambda / 2$ in length, so that a standing wave is created and the electric field is maximized in the center where the PSBs are located. The two absorbers are coaxial and are separated by only $100 \mu \mathrm{m}$ to ensure that they sample the same electric field.

The PSB modules are referenced in position and heatsunk to the back flange of the feed horns. The angular alignment of each module is set by a stainless steel dowel pin on the module and a machined radial slot in the back of the focal plane. The dominant angular alignment error is in the placement of the silicon nitride absorbing grids within the modules, which is done under a microscope. Section 7.3 reviews the characterization of the detectors, and Section 7.6 discusses the measurements of their polarization properties.

\section{CRYOGENICS AND THERMOMETRY}

\subsection{Cryostat}

The QUaD receiver is housed in a custom-made liquid helium/liquid nitrogen cryostat (shown in schematic in Figure 4) manufactured by AS Scientific. ${ }^{22}$ The instrument is upward looking, with the cold reimaging optics housed in a snout that is inserted through the hole in the center of the primary mirror. The receiver vacuum window is located on top of the snout. It is made from ultra-high molecular-weight polyethylene (UHMW PE), roughly $1 \mathrm{~cm}$ thick, and the surfaces are AR coated to improve transmission.

The focal plane and cryogenic electronics (Sections 4 and 6.1) are assembled on a removable central core that is enclosed by concentric, toroidal liquid cryogen tanks. The capacities of the tanks are $35 \mathrm{~L}$ for liquid nitrogen and $21 \mathrm{~L}$ for liquid helium, resulting in hold times of 3.5 and 1.3 days respectively, including the daily fridge cycle. During normal observation, both tanks are filled once per day.

A G-10 fiberglass truss frame supports the tanks, providing rigidity with low thermal conductivity. Sections of thin-walled

\footnotetext{
21 J. Beeman, www.haller-beeman.com

22 Abingdon, UK. www.asscientific.co.uk
} 


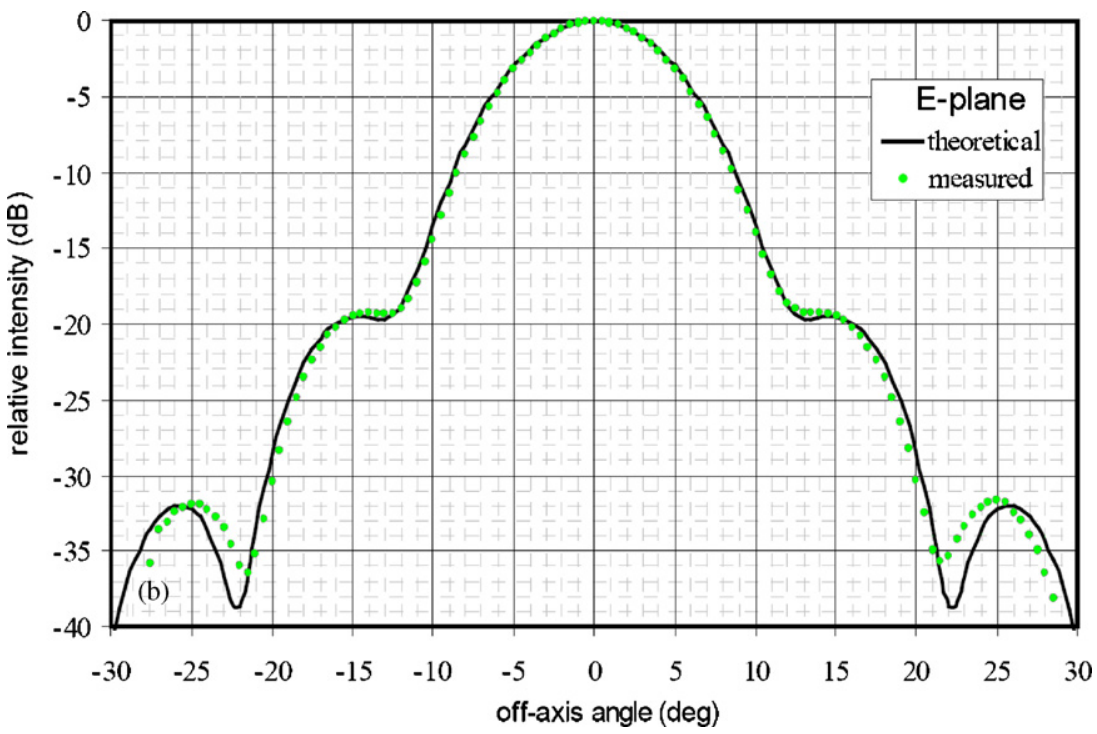

Figure 9. Measured and predicted beam pattern from a QUaD $100 \mathrm{GHz}$ corrugated feed horn. (A color version of this figure is available in the online journal.)

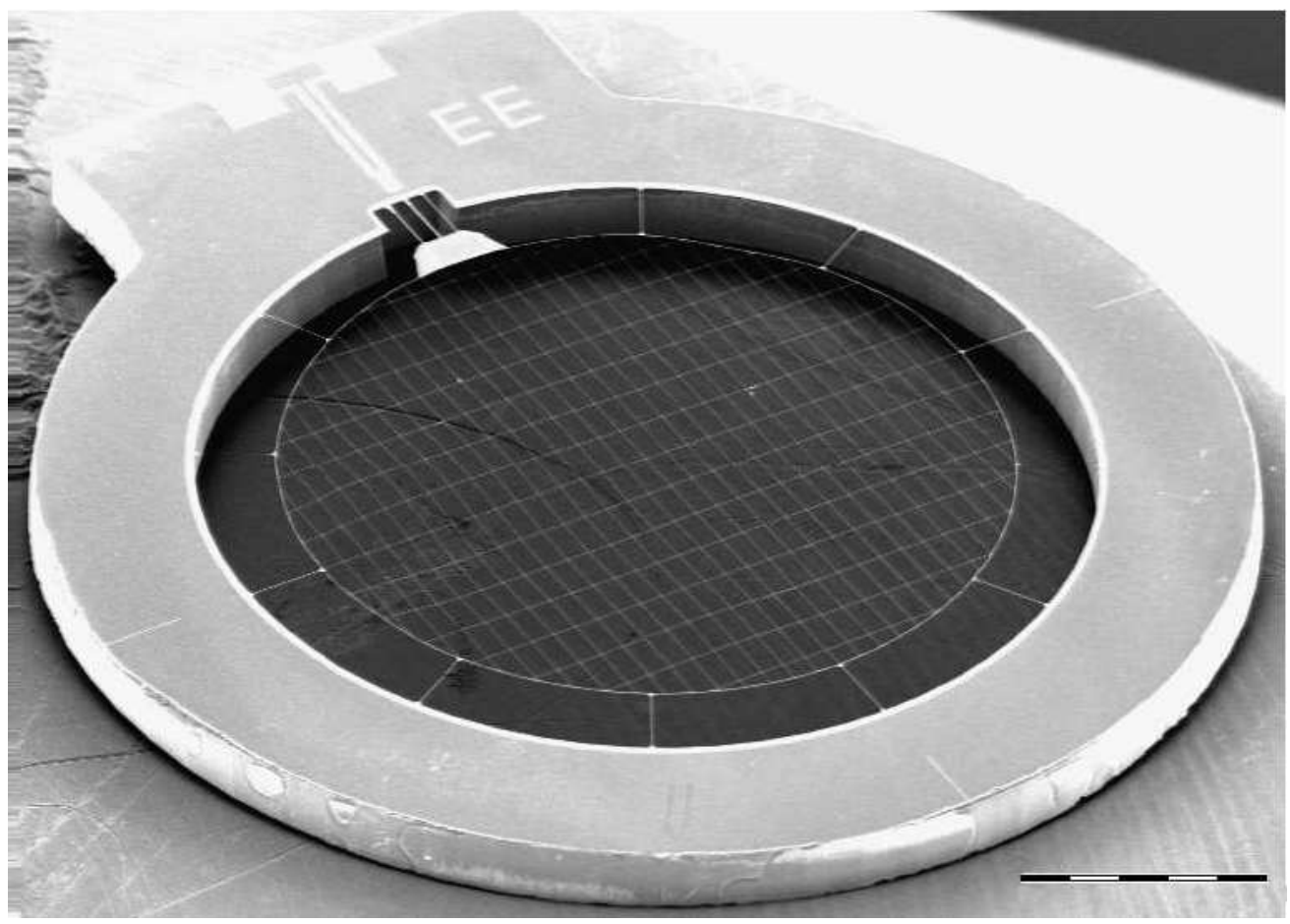

Figure 10. Scanning electron micrograph of a single PSB membrane. The $4.5 \mathrm{~mm}$ diameter absorber is suspended with an array of radial supports. Metalized traces (running from the upper left to the lower right) selectively absorb one linear polarization of incident optical radiation. Perpendicular, nonmetalized traces provide mechanical support. The $150 \mu \mathrm{m}$ spacing of the conductors allows the same membranes to be used for the $100 \mathrm{and} 150 \mathrm{GHz}$ bands. A thermistor, located on the edge of the absorber (upper left), measures the temperature. Three thicker legs (upper left) set the thermal conductance to the housing (known as $G$ ). The center leg also caries the electrical leads from the thermistor to a pair of wirebond pads. The outer two legs could be trimmed, allowing $G$ to be tuned to one of the four possible values.

stainless steel bellows limit the conductivity through the fill tubes and allow for differential contraction of the different thermal stages. The cryostat has four fill tubes (fill and vent per tank), two of which house gauges to monitor cryogen levels. Because the fill tubes are inaccessible when the cryostat is installed on the telescope, refills are performed via flexible transfer lines, about $3.5 \mathrm{~m}$ long, made out of two sections connected through standard bayonet fittings. The shorter section is inserted in the cryostat fill tube and remains in place throughout the observing season. The longer section is only connected during refills.

Within the cryostat, both liquid cryogen tanks, as well as the aluminum radiation shields thermally connected to them, are wrapped in multilayer aluminized mylar insulation. Filtered apertures on the top of each shield allow the optical signal to reach the focal plane, while blocking out-of-band IR radiation (see Figure 3). The focal plane is surrounded by an additional radiation shield that is thermally connected to the intermediate 
stage of the sub-Kelvin refrigerator, maintaining a temperature of $430 \mathrm{mK}$. The large focal plane mass $(\sim 10 \mathrm{~kg})$ combined with the thermally isolating support structure results in a long cool down period. Starting from room temperature, both tanks are initially filled with liquid nitrogen. Approximately five days later, the focal plane reaches $100 \mathrm{~K}$ at which point the inner tank is filled with liquid helium. Two more days of precooling are then required before the fridge can be cycled.

In order to speed up the cool down, two active He-3 gasgap heat switches are used. ${ }^{23}$ The first connects the fridge $4 \mathrm{~K}$ baseplate to the intermediate stage. The second connects the fridge intermediate stage to the ultracold stage. These switches are turned off once the focal plane temperature approaches $4 \mathrm{~K}$. The heat switch connected to the fridge ultracold stage has an estimated off-conductance $<0.1 \mu \mathrm{W}$.

\subsection{Fridge}

The focal plane is cooled to an operating temperature of $\sim 250 \mathrm{mK}$ using a three-stage $\mathrm{He}-4 / \mathrm{He}-3 / \mathrm{He}-3$ sorption refrigerator, manufactured by Chase Research Cryogenics (Bhatia et al. 2000). Similar fridges have been used successfully in bolometer-based receivers such as ACBAR and BOLOCAM (Runyan et al. 2003; Glenn et al. 1998). The first and second fridge stages, known together as the Intercooler (IC), contain, respectively, He-4 and He-3. The third stage contains He-3 and is referred to as the Ultracooler (UC). The fridge is cycled by first condensing $\mathrm{He}-4$ and using the enthalpy of that liquid to cool the IC and UC condensation points below the He-3 critical temperature. During normal operations at the South Pole, the fridge IC and UC stages achieve temperatures of $430 \mathrm{mK}$ and $253 \mathrm{mK}$. The heat loads on the fridge stages are measured to be $\sim 40 \mu \mathrm{W}$ on the IC and $<0.5 \mu \mathrm{W}$ on the UC.

To achieve better condensation efficiency during the cycle, the fridge is mounted directly on the LHe tank, in a position that keeps the fridge baseplate in contact with liquid helium when the telescope is at the typical observing elevation of $\sim 50^{\circ}$. To preserve this contact, rotation of the system about the optical axis ("deck" rotation) is limited to $\sim \pm 60^{\circ}$ during normal observing.

The large focal plane mass $(\sim 10 \mathrm{~kg})$ presented a particular challenge to achieving an optimized fridge cycle. A maximum possible hold time and minimum loss of observing due to the cycling time were desired. The procedure that was developed (shown in Figure 11) allowed the QUaD fridge to be cycled in approximately four hours and results in hold times for the UC and IC stages of 24 and $31 \mathrm{hr}$, respectively (duty cicle $\sim 83 \%$ ). Once the optimized cycle was determined, the procedure was automated (Section 5.4) so that the operator can perform a fridge cycle by issuing a single command on the control system.

\subsection{Receiver Core}

The receiver core comprises the focal plane and the associated mechanical and electrical support hardware (Figure 5). It is composed of three stages that are each held at a different temperature during operation: a $4 \mathrm{~K}$ baseplate, a $430 \mathrm{mK}$ intermediate stage, and the $250 \mathrm{mK}$ focal plane assembly.

The $4 \mathrm{~K}$ baseplate is made of gold-plated 6061 aluminum. It supports the rest of the focal plane structure and provides the mounting point for the two JFET amplifier boxes (Section 6). When installed in the cryostat, the baseplate is mechanically attached to the liquid helium tank through a cylindrical aluminum

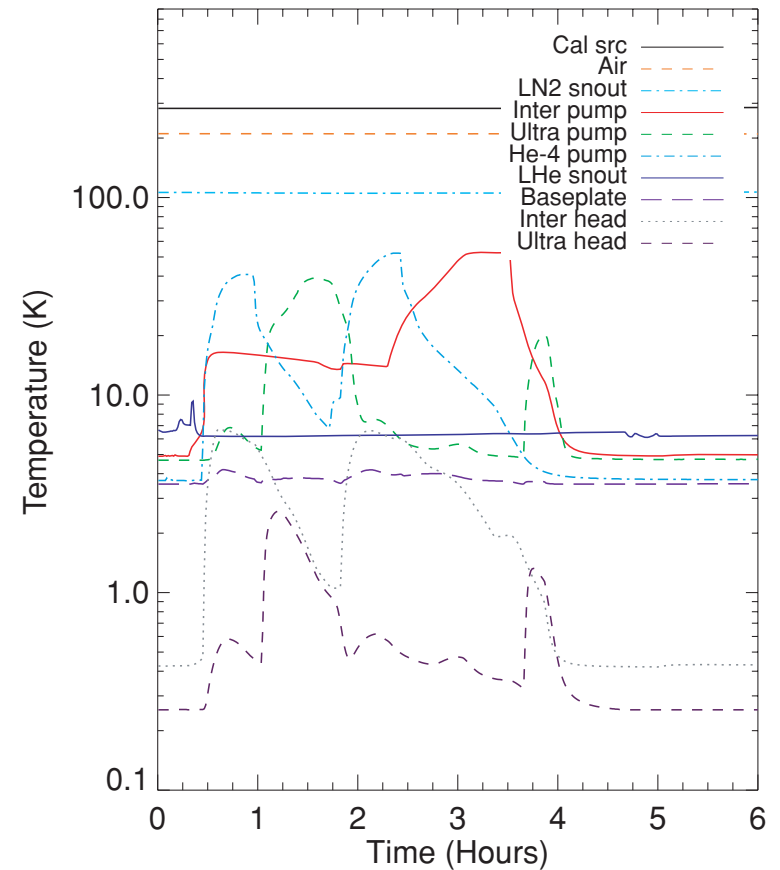

Figure 11. Temperatures of QUaD components through a fridge cycle at the start of an observing day. "Cal src" is the temperature of the calibration source located above the secondary mirror, inside the foam cone (Section 9). "Air" is the external air temperature. "LN2 snout" and "LHe snout" are the temperatures at the top of the liquid nitrogen and liquid helium cryostat snouts. The remaining items are the temperatures of the fridge pumps, cold heads, and baseplate (Section 5.2)

(A color version of this figure is available in the online journal.)

radiation shield. The dominant thermal path is through two parallel OFHC copper straps that connect to the helium tank. This ensures that the baseplate stays near $4 \mathrm{~K}$ despite the $26 \mathrm{~mW}$ dissipation from the JFETs.

The intermediate stage is a gold-plated aluminum ring that is thermally connected to the fridge IC. It is mechanically attached to the $4 \mathrm{~K}$ baseplate by a six-legged hexapod structure of 6" SP-1 Vespel tubes (0'.438 outside diameter and 0'031 wall thickness). The focal plane assembly is mounted to the intermediate stage with six shorter legs made from SP-22 Vespel $^{24}$ tubing machined to the same diameter and wall thickness. The focal plane is thermally connected to the fridge UC.

The estimated heat load on the fridge from the Vespel supports is $0.13 \mu \mathrm{K}$ on the UC and $24 \mu \mathrm{K}$ on the IC. SP-22 is used for the focal plane supports because it has lower thermal conductivity than SP-1 in the sub-Kelvin temperature range (Runyan 2002). Both the focal plane and the intermediate stage are attached to their respective fridge cold heads via flexible, copper heat straps made from braided OFHC copper electrical shielding. Multiple braids are twisted together to increase the cross-sectional area and the straps are annealed for increased conductivity.

Because of the high electrical impedance of the bolometers, microphonic pickup from vibrations of the wiring is a concern (Section 8). For this reason, all of the wiring between the JFET amplifiers and the bolometers must be rigidly supported. The Vespel legs that support the focal plane provide natural attachment points for the wiring that runs between the stages (Figure 5). All of the nonisothermal wires in the cryostat are manganin (0'.003) ribbon cables woven into a robust ribbon 
cable with nylon thread for strain relief. ${ }^{25}$ The wiring is tightly wrapped around the Vespel legs along a helical path and fixed down at regular intervals using a combination of teflon tape and lacing tape. Two additional Vespel tubes between the intermediate stage and the JFET modules act as a bridge to support the wiring along this critical signal path. The heat load on the fridge UC stage is reduced by heat sinking the focal plane wiring to the intermediate temperature stage. The heat load from the wiring is estimated to be $\sim 8 \mu \mathrm{W}$ on the fridge IC and $<0.1 \mu \mathrm{W}$ on the UC. On the isothermal stages, including the back of the focal plane bowl, stiffer (28 AWG) copper wiring is used and is held down by the aluminum tape. Specially designed aluminum brackets support the wiring at all connector interfaces, including the PSB modules.

\subsection{Thermometry and Temperature Control}

The temperatures of all the major cryogenic components are monitored, including the focal plane, the fridge pumps, cold heads, and baseplate, the snout that holds the two lenses, and the liquid cryogen tanks. Silicon diode sensors ${ }^{26}$ monitor the temperatures of components that operate at or above $4 \mathrm{~K}$. Germanium resistance thermometers ${ }^{27}$ (GRTs) monitor the temperatures of sub-Kelvin components. The diodes that are mounted on the focal plane allow the monitoring of the cool down from room temperature; however, their large power dissipation $(\sim 15 \mu \mathrm{W})$ requires that they be switched off during sub-Kelvin operation.

Figure 11 shows temperature readouts during and shortly after a routine fridge cycle. In order to monitor the temperatures of all the different cryogenic components and to operate the heaters of fridge pumps and gas-gap heat switches, required to cycle the sorption fridge, we use custom-made thermometer readout and control electronics. With a very compact design (only one main unit and one separate power supply unit), it can readout up to six GRTs and 21 diodes, and operate five heaters. An embedded computer, the $\mathrm{TINI}^{28}$ (Tiny INternet Interface), reads the digitized voltage outputs, sets the heaters drives, and communicates with the control system. With this system it is possible to cycle the fridge with no external intervention, by running a script on the TINI. The same electronics drives two additional heaters (with constant drive and manual control) for the gas-gap heat switches we use to precool fridge and focal plane at liquid helium temperature.

The focal plane temperature is stabilized with a separate system. Temperature is read out with a custom AC bridge connected to an NTD Ge thermistor (Haller-Beeman) located on the focal plane. The bridge operates at the bolometer bias frequency. A Stanford Research Systems SIM960 PID controller, served off the thermistor readout, drives three heater resistors in parallel, located symmetrically around the joint between the fridge heat strap and the focal plane. An additional thermistor, read out with the same system, is used as a temperature monitor. During routine observing, the PID set point is $258 \mathrm{mK}, \sim 5 \mathrm{mK}$ above the natural operating temperature of the fridge, requiring $\sim 0.3 \mu \mathrm{W}$ of electrical heater power. This maintains a stable temperature despite the telescope motion associated with raster scanning and the slight warming of the fridge UC that occurs over the course of an $18 \mathrm{hr}$ observation.

\footnotetext{
25 www.tekdata.co.uk

26 Lake Shore DT-470 series, Lake Shore Cryotronics, Inc., www.lakeshore.com

27 Lake Shore GR-200 series

28 MAXIM, www.maxim-ic.com
}

Table 2

Electronics Parameters

\begin{tabular}{|c|c|}
\hline Number of channels & 96 \\
\hline DC mode gain & 200 \\
\hline AC mode gain & $10^{5}$ \\
\hline Capacitance $^{\mathrm{a}}(\mathrm{pF})$ & 85 \\
\hline Load resistance $(\mathrm{M} \Omega)$ & 40 \\
\hline Bias generator noise $\left(\mathrm{nV} \mathrm{Hz}^{-1 / 2}\right)$ & 3 \\
\hline JFET noise $\left(\mathrm{nV} \mathrm{Hz}^{-1 / 2}\right)$ & 7 \\
\hline Warm amplifier noise $\left(\mathrm{nV} \mathrm{Hz}^{-1 / 2}\right)$ & 5 \\
\hline Total electronics noise $\left(\mathrm{nV} \mathrm{Hz}^{-1 / 2}\right)$ & 9 \\
\hline $1 / f$ knee $(\mathrm{mHz})$ & 10 \\
\hline $\mathrm{AC}$ bias frequency ${ }^{\mathrm{b}}(\mathrm{Hz})$ & 110 \\
\hline $\mathrm{AC}$ bias current ${ }^{\mathrm{c}}(\mathrm{nA})$ & 1.25 \\
\hline Readout bandwidth (Hz) & 20 \\
\hline Sampling frequency $(\mathrm{Hz})$ & 100 \\
\hline $\operatorname{Power}^{\mathrm{d}}(\mathrm{W})$ & 60 \\
\hline
\end{tabular}

Notes.

a The capacitance between the two signal leads of a channel arising from the connectors and wiring between the PSBs and the JFETs.

$\mathrm{b}$ The bias frequency is adjustable from 40 to $250 \mathrm{~Hz}$. The frequency is chosen to minimize interference (Section 8).

c The bias current is adjustable from 0 to $30 \mathrm{nA}$. The current is a tradeoff between stability and sensitivity (Section 7).

d Power for the amplifier boxes, not including the commercial ADC system.

\section{ELECTRONICS}

The QUaD electronics employ an AC-biased, fully differential readout to amplify the bolometer signals. The basic scheme has a long history in bolometric CMB instruments (see Holzapfel et al. 1997; Glenn et al. 1998; Crill et al. 2003, for instance). An overview of the electronics chain is shown in Figure 12. The bias generator excites the bolometers with a sinusoidal current through a pair of equal-valued load resistors. The balanced nature of the bolometer/load resistor bridge minimizes crosstalk and pickup along the high-impedance wiring leading to the cryogenic JFET buffer amplifiers. The warm electronics amplify, demodulate, and filter the output from the JFETs. Finally, the data acquisition system digitizes and archives the processed signals. The QUaD electronics are interfaced to the control software to allow the bias frequency and amplitude, amplifier gain, DC offset removal, and phase adjustment to be set remotely. Table 2 gives the parameters of the readout electronics chain. The electronics development and testing are described in more detail in Hinderks (2005).

\subsection{Cold Electronics}

The load resistors are Nichrome metal film on a silicon substrate in a surface-mount package. ${ }^{29}$ There are two $20 \mathrm{M} \Omega$ load resistors in each circuit. This value was chosen to be much larger than the bolometer resistance to provide a bias current that remains approximately constant for small loading changes. The load resistor boards (Figure 13) are located on the $250 \mathrm{mK}$ stage to reduce Johnson noise. The boards were fabricated from standard 1/16" FR-4 substrate. Input and output from the board is via surface-mount 51-way micro-D connectors. ${ }^{30}$ These connectors have leads that were specifically designed to take up differential contraction between the fiberglass board and the aluminum mounting structure that is used to provide

\footnotetext{
29 Mini Systems, Inc., www.mini-systems.biz

30 Cristek Interconnects Inc., www.cristek.com
}

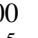 . 3 7 9 .

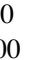 .}

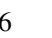

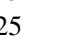




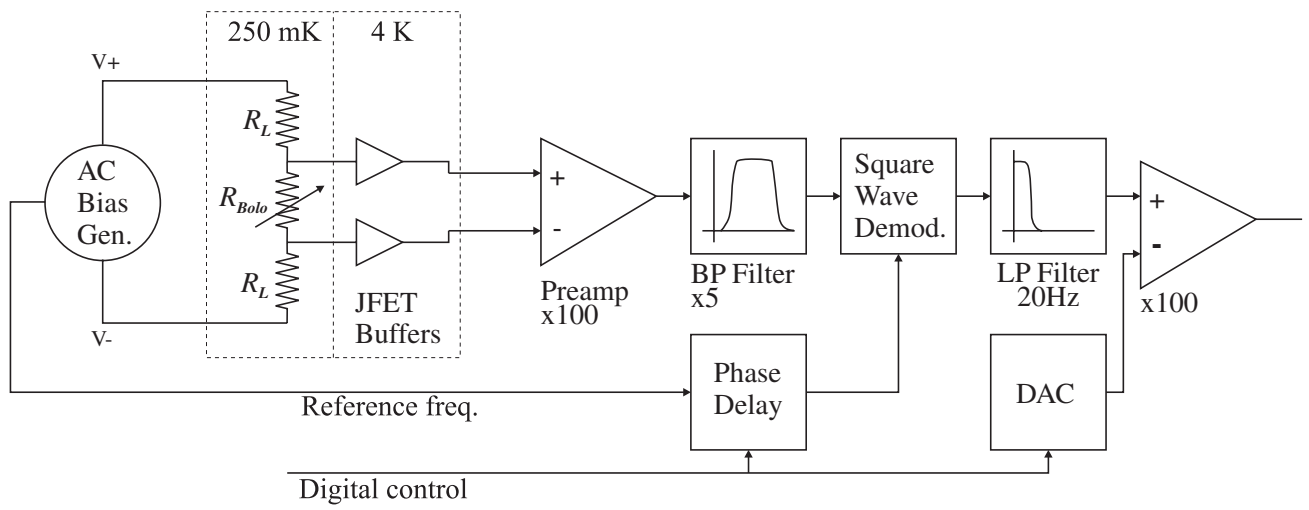

Figure 12. Block diagram of the QUaD readout electronics. The components within the dashed lines are located in the cryostat. The load resistors and detectors are on the focal plane assembly and operate at approximately $250 \mathrm{mK}$. The JFET amplifiers modules are mounted on the $4 \mathrm{~K}$ baseplate; however, within the modules the JFETs themselves operate at roughly $120 \mathrm{~K}$ (Section 6).

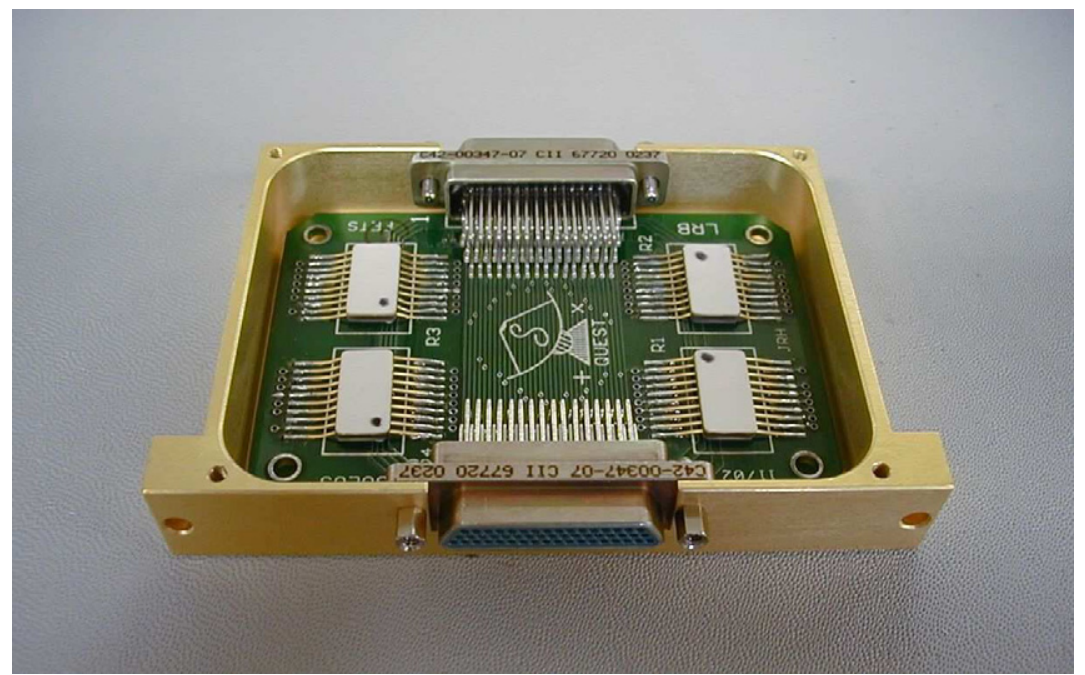

Figure 13. Photograph of a QUaD load resistor board. Each board contains 56 resistors (including eight spares) in four surface-mount packages and services up to 24 channels. The boards themselves are with double-sided 1 oz copper and 8 mil traces. Input and output from the board is via surface-mount 51-way micro-D connectors (Cristek Interconnects Inc., www.cristek.com). These connectors have long surface-mount leads that take up differential contraction between the fiberglass board and the aluminum mounting structure that provides mechanical support.

(A color version of this figure is available in the online journal.)

mechanical support. In three years of sub-Kelvin operation and numerous thermal cycles, we did not experience a single failure.

The JFET amplifiers are located in two boxes mounted on the $4 \mathrm{~K}$ baseplate, directly below the focal plane assembly. The transistors themselves operate at an elevated temperature of $\sim 120 \mathrm{~K}$ for optimum noise performance and consequently need to be thermally isolated from their $4 \mathrm{~K}$ enclosure. Each box contains two silicon nitride membranes, each holding 24 dual JFET dies ${ }^{31}$ allowing readout of 48 channels per box. The membranes provide thermal isolation of the amplifiers from the $4 \mathrm{~K}$ stage and are mechanically supported by the micro-D input/output connectors. The JFETs are configured as source followers with their drains connected to a common positive supply and the sources connected via $125 \mathrm{k} \Omega$ resistors to the common negative supply. The power supply voltages for each membrane is adjusted for lowest noise performance. Each membrane dissipates $\sim 6.5 \mathrm{~mW}$ during normal operation with heater resistors used to assist at startup. The low thermal mass allows the membranes to reach a stable operating temperature in approximately one minute.

\footnotetext{
31 Siliconix U401
}

\subsection{Warm Electronics}

The bias generator is used to provide a stable sine-wave excitation that can be adjusted over a frequency range of $40-250 \mathrm{~Hz}$ with 10 bit resolution. The sine wave is generated by filtering a square wave with a $Q=10$ bandpass filter. A low-noise DC voltage reference and an analog modulator switched by a crystal oscillator generate the square wave. The bandpass filter is made electronically tunable by using multiplying DACs in place of fixed resistors. The bias frequency is common to all bolometers but the $100 \mathrm{GHz}$ and $150 \mathrm{GHz}$ bias amplitudes can be independently adjusted. A DC bias mode is provided for testing and is used for taking bolometer I-V curves (Section 7.3). The bias amplitudes are archived along with the bolometer data. The bias frequency, amplitude and bandpass tuning are computer controlled via a serial interface.

The bolometer signals are amplified with low-noise AD624 amplifiers and then filtered with a broad bandpass filter $(Q \sim 0.5)$ that is designed to have a close to flat response over the available range of bias frequencies. The signals are then multiplied by a square-wave reference from the bias board to demodulate the component at the bias frequency. An adjustable delay circuit for each channel compensates the demodulator 
reference signal for phase shifts within the cryostat. A steep low-pass filter $(20 \mathrm{~Hz}, 6$-pole) follows the demodulator.

To ease the dynamic range requirement on the ADC system, a 12-bit DAC is adjusted periodically to null out the large DC component of the demodulated signal. This is followed by an additional gain stage of 100. During observation, the DC-offset removal DAC settings are adjusted approximately every 30 minutes to prevent any channels saturating due to $1 / f$-noise or elevation changes.

The electronics can be switched to a "low-gain" mode in which the DC-offset removal and additional gain stage are bypassed. This mode is useful for testing, and is used routinely by the control system to automatically determine the appropriate setting for each channel's DC-offset removal DAC. Finally, buffer circuitry creates a balanced differential output for driving the cabling to the ADC. All of the settings including reference phase delay, DC offset removal, and gain mode are controlled via a serial interface.

The bias and readout electronics are located in radio frequency (RF) boxes attached via an RF-tight interface box directly to the bottom surface of the cryostat. All wiring entering the cryostat passes through these interface boxes and is RF filtered using filtered D-sub connectors. ${ }^{32}$

\subsection{Data Acquisition and Control System}

Real-time operations including telescope control, and digitization of the bolometer data, are handled by a VME controller running VXWorks in a crate mounted adjacent to the cryostat. Two 64-channel, 16-bit ADC cards digitize the bolometer data. A 32-channel DIO card provides the control interface to the bias and readout boards. A Linux-based PC system provides the user interface and data archiving for the real-time controller.

Once per second, the software archives a complete snapshot of the system, known as a register frame. Each register frame contains the most recent values from all of the attached hardware, including thermometry, bias settings, and DC offset removal values. The bolometer data along with the telescope axis encoders are sampled at $100 \mathrm{~Hz}$, with 100 samples per channel stored in each register frame. Software commands are provided for controlling all the components of the system. These commands are combined to form complete observing scripts. A client program allows users to run scripts and monitor the data in real time.

\section{INSTRUMENT CHARACTERIZATION}

\subsection{Telescope Pointing}

QUaD used a nine-parameter pointing model with the telescope control computer handling full conversion from right ascension/declination/paralactic angle request values to azimuth/elevation/deck encoder command values. The model parameters were established from a combination of optical data from a small telescope attached to the elevation structure of the mount and from several special day-long radio pointing runs. These radio pointing runs consisted of scanning the central pixel across five bright, compact millimeter sources (MAT6A, NGC3576, IRAS1022, RCW38, and IRAS08576) in both azimuth and elevation, in a pattern known as a "pointing cross." This determines the pointing offset between the optical and radio systems, the flexure with elevation of the radio pointing, and

\footnotetext{
$\overline{32}$ Spectrum Control Series 700 with $1000 \mathrm{pF}$ PI filters.
}
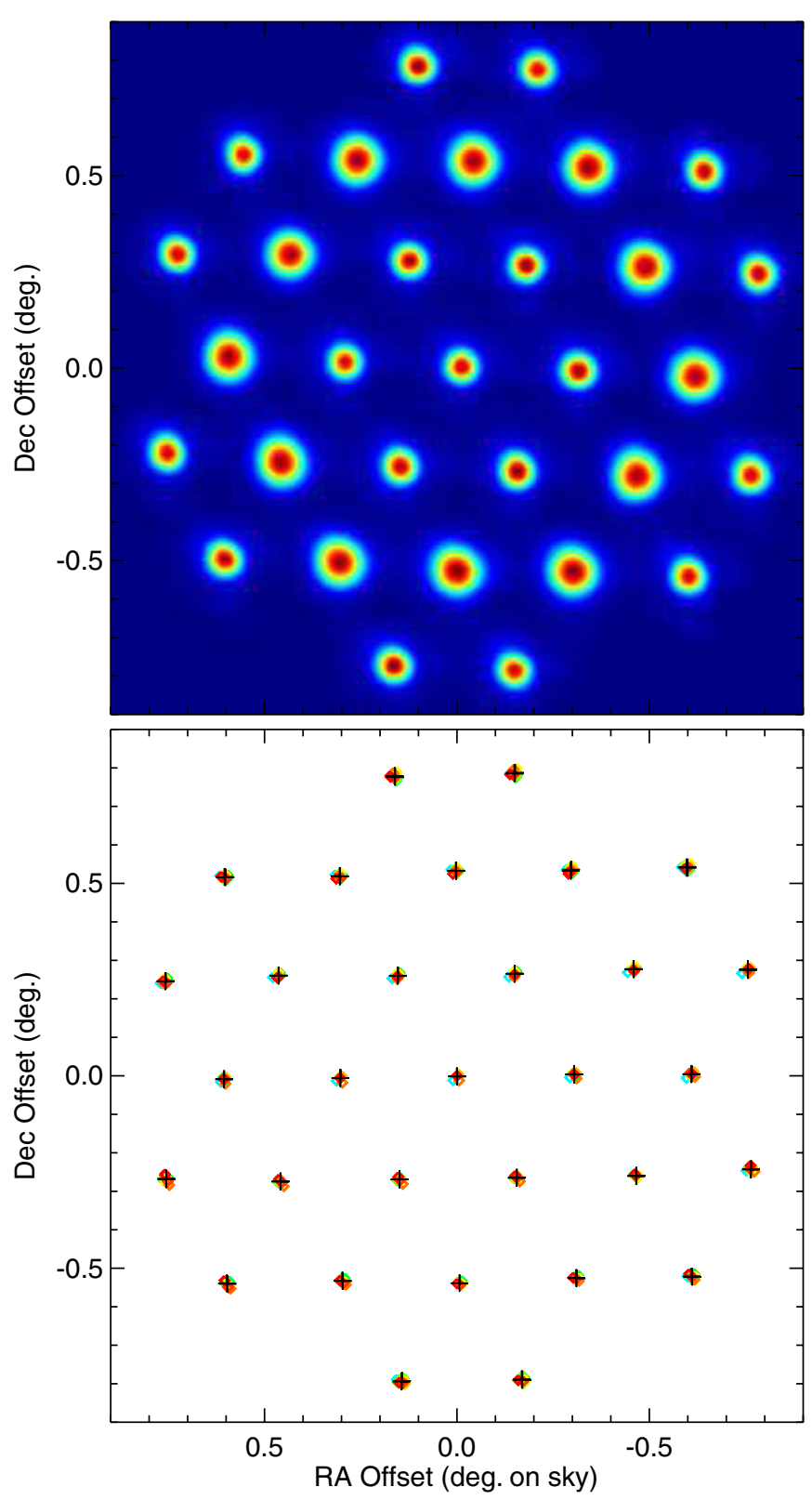

Figure 14. Top: raster map over the galactic source RCW38. The map has been smoothed with a 1'2 Gaussian. Bottom: radio pointing locations for each channel derived from 12 full raster maps spread over the second and third seasons. The black crosses indicate the mean offset for each channel.

(A color version of this figure is available in the online journal.)

the magnitude and angle of the offset between the radio pointing direction and the rotation axis of the mount's third axis. The azimuth axis tilts and encoder zero points were measured a few times per season from the optical data. Later radio pointing runs established the accuracy of the absolute pointing at $\sim 0.5$.

Approximately one day per month was devoted to making full raster maps using a bright source, usually RCW38 (Figure 14, top). Gaussian profiles were fitted to the raster data and were then used to determine the pointing offset of each feed. The feed offsets obtained from 12 full beam maps are shown in Figure 14 (bottom). The offset angles used in the data analysis were determined by averaging over the 12 mapping runs, to reduce the effects of the random pointing wander that occurs during each $16 \mathrm{hr}$ run. The rms scatter in the feed offset angles versus the mean is $\sim 0.3$ (Figure 15). There is no evidence for a systematic change in the feed offset angles over time. 


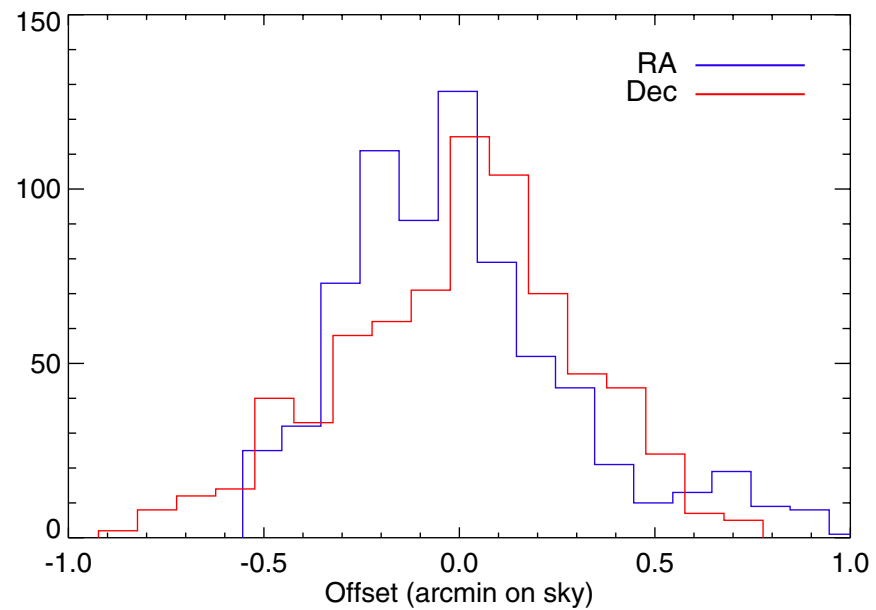

Figure 15. Histogram showing the variation in the derived radio pointing for all feeds for seasons 2 and 3, from the data plotted in the bottom panel of Figure 14. The rms of both distributions is $\sim 0.3$.

(A color version of this figure is available in the online journal.)

In addition to the monthly raster scans in which the entire focal plane was mapped, pointing checks were performed every $8 \mathrm{hr}$ during routine observations with a pointing cross observation on RCW38 (Figure 16). The scatter of the derived pointing from the cross observations is $\sim 0.4$ (also in Figure 16) which provides a useful cross-check on the pointing uncertainty derived from the full focal plane raster scans. Attempts were made to use these offsets to make pointing corrections during offline data analysis but it was not possible to demonstrate any clear improvement using this method.

Furthermore, in a procedure known as the "row-cal," each of the seven rows of the array is scanned in turn across RCW38 in the azimuth. Fitting one-dimensional Gaussian profiles to the RCW38 blips in the row-cal time-ordered data of each channel allows us to monitor the relative angular separations between feeds in a given row. Figure 17 shows the separations between pairs of adjacent feeds measured from one row-cal per day. The scatter in these measurements is less than 0.1 with little drift, confirming the long-term stability of the array.

\subsection{Beam Characterization}

Because of the geographic location, and the elevation limit imposed by the ground shield, QUaD is not able to view planets. As they are very bright and compact, they are the preferred targets for beam mapping at millimeter wavelengths. RCW38, which is the brightest of the compact H II regions visible to QUaD, has a $\sim 1^{\prime}$ intrinsic width (Coble et al. 2003, and our own measurements) and rich extended structure that makes it difficult to use for absolute determination of beam parameters. Because of this, the QSO PKS 0537-441 (WMAP source PMN J0538-4405) was used for this purpose. It is a true point source, but at only $\sim 5 \mathrm{Jy}$, compared to the $145 \mathrm{Jy}$ flux of RCW38 at $150 \mathrm{GHz}$, the quasar observations required significantly more integration time than was used for the RCW38 raster maps. Figure 18 shows a quasar beam map obtained from 3 days of observation, averaged over all detectors used for the final CMB maps. The beam widths used in the Data Paper $(5.0$ at $100 \mathrm{GHz}$ and 3.5 at 150) are based entirely on maps of this quasar. There is evidence for small channel-to-channel variations in width, and for ellipticity $<10 \%$.

In the first season of observation, as shown in Figure 19, the beams had significant ellipticity that was found to vary with external temperature as the foam cone supporting the secondary expanded and contracted. The ellipticity was traced to a small saddle-shaped warp of the primary mirror. The shape of the primary was accurately measured after installation on the QUaD mount, using an articulated measurement arm. ${ }^{33}$ The measured warp has an angular dependence of $\cos (2 \theta)$ and an amplitude of approximately $0.25 \mathrm{~mm}$ at the outer edge of the mirror (where the beam intensity is down by a factor of 100).

The beam shapes measured in season one can be accurately described by an optics model that included the measured warp, and in the first season this model was used to determine the secondary mirror position that minimized the average ellipticity across the focal plane. In the summer following the first season, a shaped secondary mirror was installed that corrected for the primary, providing nearly symmetric beams for the second and third season's observations. The optics modeling is described in more detail in O'Sullivan et al. (2008).

Analysis of the row-cal scans of RCW38 from the first season showed that the beam shape varied with the external air temperature. A beam model, derived from the row-cal data, was used to correct for this effect. For the second and third seasons, with the shaped secondary mirror installed, a similar analysis showed no significant variation with temperature or time. The beams have long-term stability well within the overall uncertainty and no correction for time variation is applied (Figure 20).

Two polarization-specific systematics are present in the QUaD beams. The first, known as squint, is an offset in the centroids of the beams from the two orthogonal detectors in a PSB pair. The effect is most severe for the outer ring of $150 \mathrm{GHz}$ pixels, with a maximum offset of 0.2 . It is stable over time and is dealt with during analysis by using the measured offsets for each PSB during simulation runs. The second effect is a mismatch in the beam shapes between the two beams of a PSB pair. Such effects can cause temperature anisotropies to appear as false polarization signals. However, simulations show that at QUaD's sensitivity, the measured level of beam mismatch is insignificant. There are several possible causes for these systematics, including birefringence or differential reflections at the lenses, multipath through the AR coatings, or polarization-dependent interactions with edges of optical elements. Further study is underway to elucidate these effects. For more detail on pointing and beam shape measurements see Zemcov (2006).

\subsection{Bolometer Parameters}

Bolometers detect incident optical power by measuring the temperature increase of an absorber that is in weak thermal contact with a fixed temperature bath. In the $\mathrm{QUaD}$ detectors, the absorber temperature is measured with an NTD Germanium thermistor that has a temperature-dependent resistance given by $R(T)=R_{0} \exp \sqrt{\Delta / T}$. The thermal conductivity between the absorber and the bath is well described by a power law $G(T)=$ $G_{0}\left(T / T_{0}\right)^{\beta}$, where $T_{0}$ is an arbitrary reference temperature, here taken to be $300 \mathrm{mK}$. Knowledge of these parameters is useful to convert measured bolometer voltages into values of total absorbed optical power by solving the power balance equation

$$
P+Q=\int_{T_{\text {bolo }}}^{T_{\text {bath }}} G(T) d T,
$$

which states that in thermal equilibrium the sum of the electrical bias power, $P$, and the optical power, $Q$, is equal to the power

\footnotetext{
33 Romer Series 3000i, www.romer.com
} 

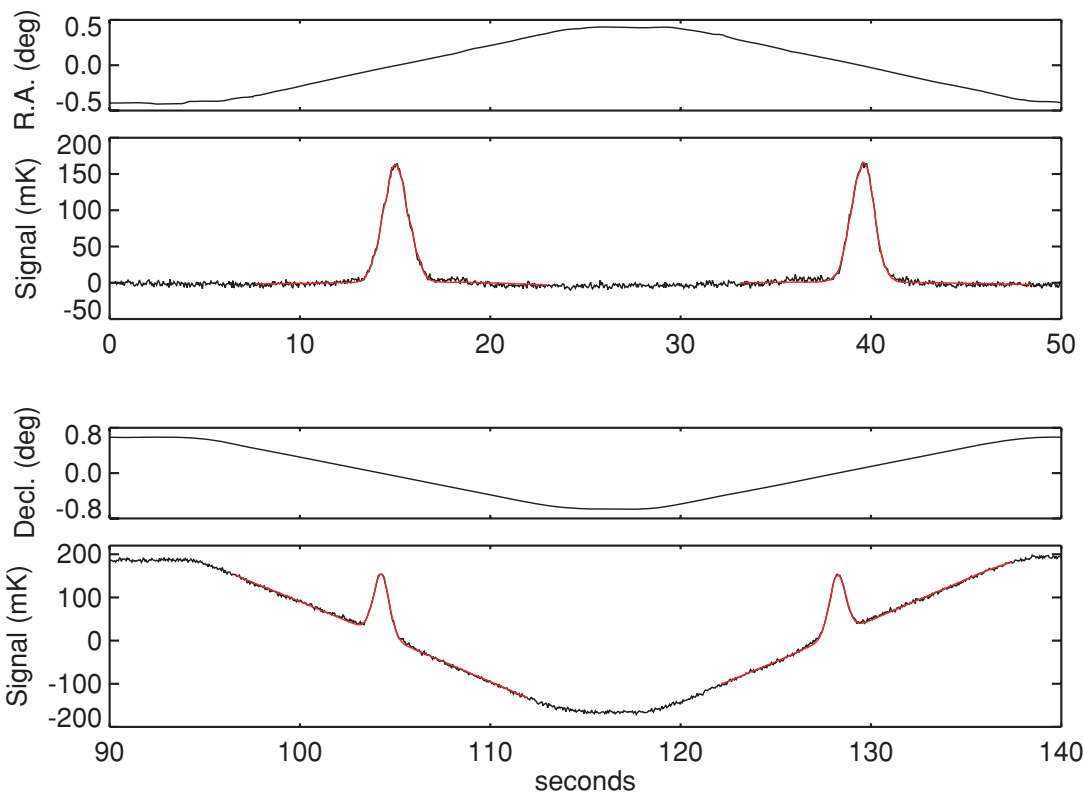

R.A.

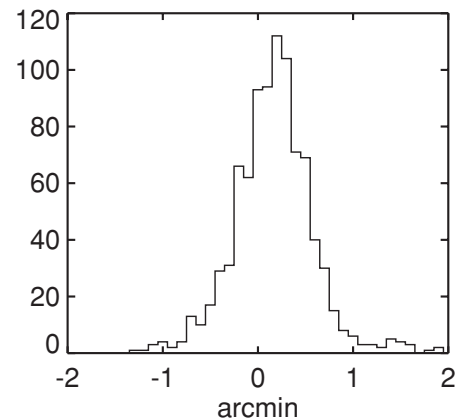

Decl.

Figure 16. Two upper panels show a "pointing cross" observation in which the central pixel is scanned across a bright source, first in right ascension (R.A.), then in declination (decl.). The lower panels show the variation of the derived pointing offset over the course of a season. The rms of both distributions is $\sim 0$ '. 4 .

(A color version of this figure is available in the online journal.)

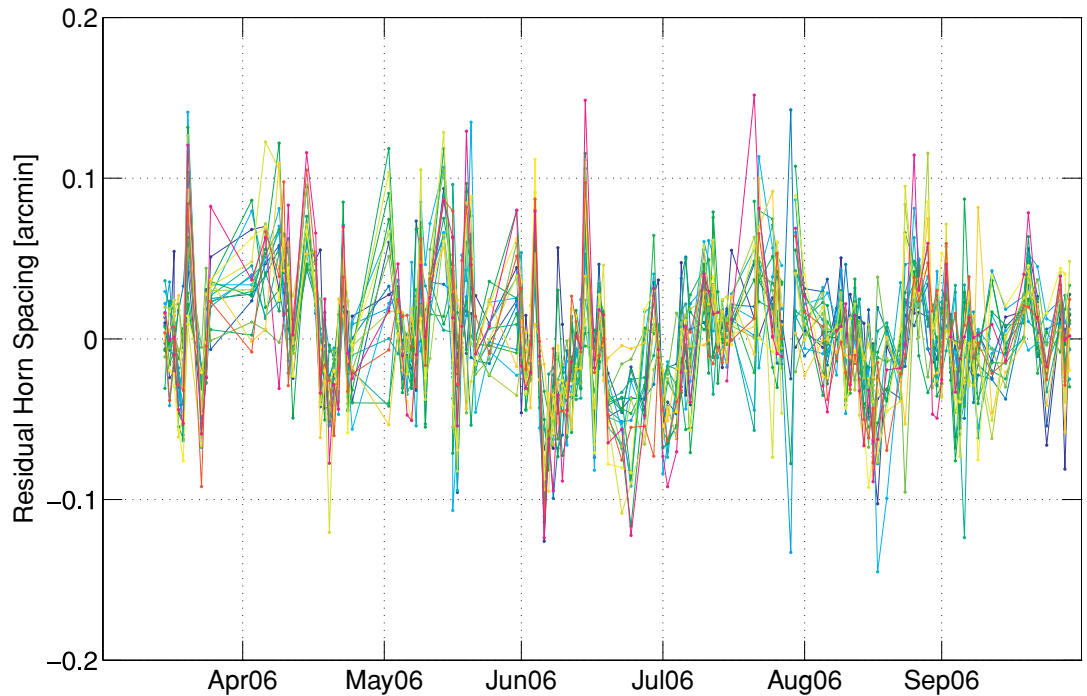

Figure 17. Residual separation between pairs of adjacent feeds as measured by daily azimuth "row-cal" scans of all channels across RCW38. The nominal feed separation is $18^{\prime}$. These daily scans are also performed with the telescope rotated by $60^{\circ}$ about the optical axis with similar results.

(A color version of this figure is available in the online journal.)

flowing from the absorber (at $T_{\text {bolo }}$ ) to the bath across the weak thermal link, $G$. It should be emphasized that this analysis is not needed to interpret the astronomical data, because during normal observation the bolometers are biased so that small changes in 

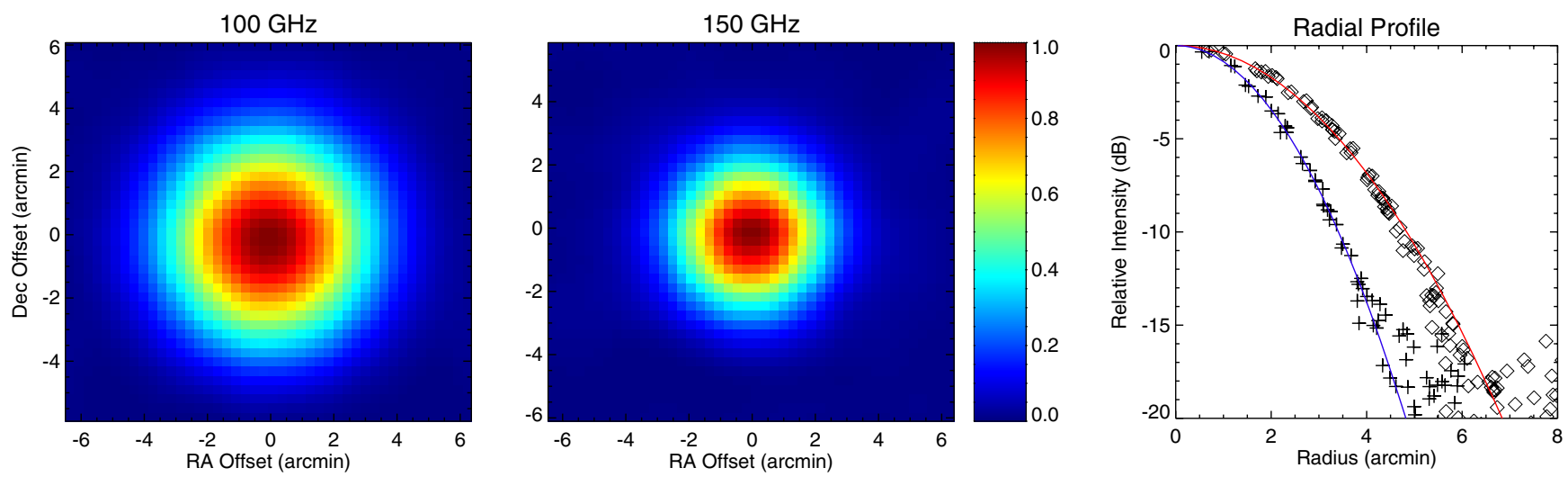

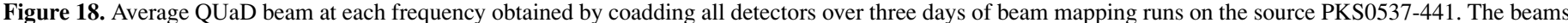
have been smoothed with a 1'2 FWHM Gaussian. Right: radial profile of the unsmoothed data to the left and the best-fit Gaussians. (A color version of this figure is available in the online journal.)
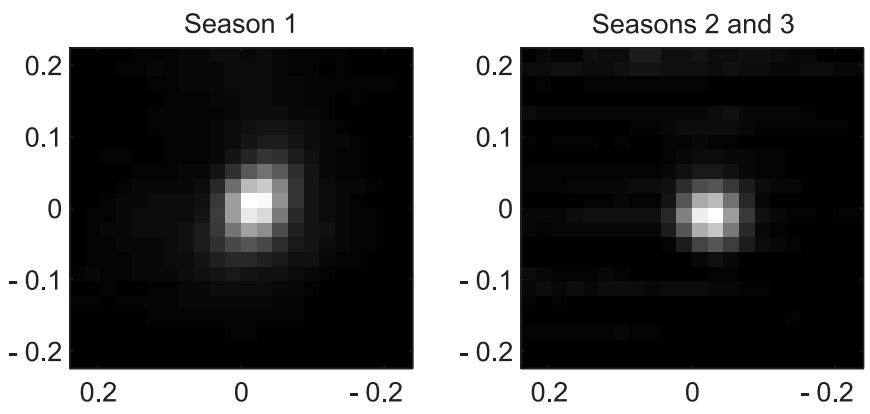

Figure 19. Single-channel beam maps from RCW38. Left: a warp in the primary mirror made the beams elliptical during the first season. Right: a shaped secondary mirror was installed for the second and third seasons, reducing the average ellipticity to $\sim 5 \%$. The axis units are in degrees.

incident optical power result in proportional changes in signal voltage as in Equation (5).

The bolometer parameters can be determined from a series of bolometer voltage versus bias current measurements, known as load curves (Figure 21), a procedure that is well documented in the literature (Sudiwala et al. 2002). Dark load curves, taken when the bolometers are blanked off, are particularly valuable for this process. Some of the QUaD detectors were tested dark in a separate cryostat prior to installation. All the detectors were dark tested with a special cool down in the QUaD cryostat during the Austral summer between the first and second observing seasons. The QUaD detectors were found to have typical values of $R_{0}, \Delta, G_{0}, \beta$ of $94 \Omega, 42 \mathrm{~K}, 120 \mathrm{pW}$ $\mathrm{K}^{-1}$, and 1.4 , respectively.

As discussed in Section 7.7, increased optical loading warms the bolometers, causing the receiver gain to fluctuate in response to changes in elevation or atmospheric conditions. In order to increase the gain stability, Germanium bolometers are usually operated at a bias that is somewhat higher than the most sensitive setting. For this reason, the QUaD bolometer biases were set at $\sim 1.2 \mathrm{nA}$, which is $\sim 40 \%$ larger than the bias setting that would give the lowest noise equivalent power (NEP; see Section 10). This results in a roughly $30 \%$ increase in gain stability versus optical loading with only a $\sim 3 \%$ increase in NEP. The typical DC responsivity at this bias setting is $\sim 3 \times 10^{8} \mathrm{~V} \mathrm{~W}^{-1}$.

\subsection{Spectral Bandpass and Optical Efficiency}

The spectral bandpass was measured for each channel using an FTS. Figure 7 shows the average spectral bands for the two frequencies. The band center is computed as

$$
v_{0}=\frac{\int v f(v) d v}{\int f(v) d v},
$$

where $f(v)$ is the unnormalized transmission function as measured by the FTS. The bandwidth is computed as the separation of the half-power points in the transmission spectra. The average measured center frequencies for the two bands are $149.5 \pm 0.5 \mathrm{GHz}$ and $94.6 \pm 0.6$, respectively, where the error indicates the standard deviation of the values for all operating channels of the band. The average bandwidths are $41 \pm 2 \mathrm{GHz}$ and $26 \pm 2$ for the 150 and $100 \mathrm{GHz}$ bands, respectively.

A bolometric receiver that is background limited has a sensitivity that is inversely proportional to the square root of the optical efficiency. The optical efficiency of the $\mathrm{QUaD}$ receiver was determined from load curves taken during laboratory observations of beam-filling blackbody loads made from unpainted Eccosorb CV-3 absorbing foam. The measurement entails recording load curves with the instrument looking at a room temperature load and then at a liquid nitrogen load. The additional electrical bias power needed to warm a detector which is observing the cold load to the temperature it was at when observing the ambient load, gives the difference in optical power actually received from these two sources. A comparison with the expected difference in optical power for a perfect system with an optical efficiency of $100 \%$ gives the optical efficiency of each detector. The mean receiver efficiency was measured to be $34 \% \pm 6 \%$ at $150 \mathrm{GHz}$ and $27 \% \pm 4 \%$ at $100 \mathrm{GHz}$ where the error again indicates the in-band channel-to-channel scatter.

\subsection{Detector Time Constants and Transfer Function}

The system transfer function must be well understood in order to convert measured detector voltage time streams into maps of intensity on the sky. For QUaD, the overall transfer function is the product of the bolometer response, $H_{b}(\omega)$, and the readout electronics response, $H_{e}(\omega)$. The electronics transfer function can be calculated from the circuit design and is given by

$$
\begin{aligned}
& H_{e}(\omega)=H_{1}(\omega) \cdot H_{2}(\omega) \\
& H_{1}(\omega)=\frac{1}{\left(s_{1}^{2}+A s_{1}+1\right)\left(s_{1}^{2}+\sqrt{2} s_{1}+1\right)\left(s_{1}^{2}+s_{1} / A+1\right)} \\
& H_{2}(\omega)=\frac{1}{\left(s_{2}^{2}+\sqrt{2} s_{2}+1\right)},
\end{aligned}
$$

where $A=-2 \cos (7 \pi / 12), s=i \omega /\left(2 \pi f_{3 \mathrm{~dB}}\right)$ and the $3 \mathrm{~dB}$ 


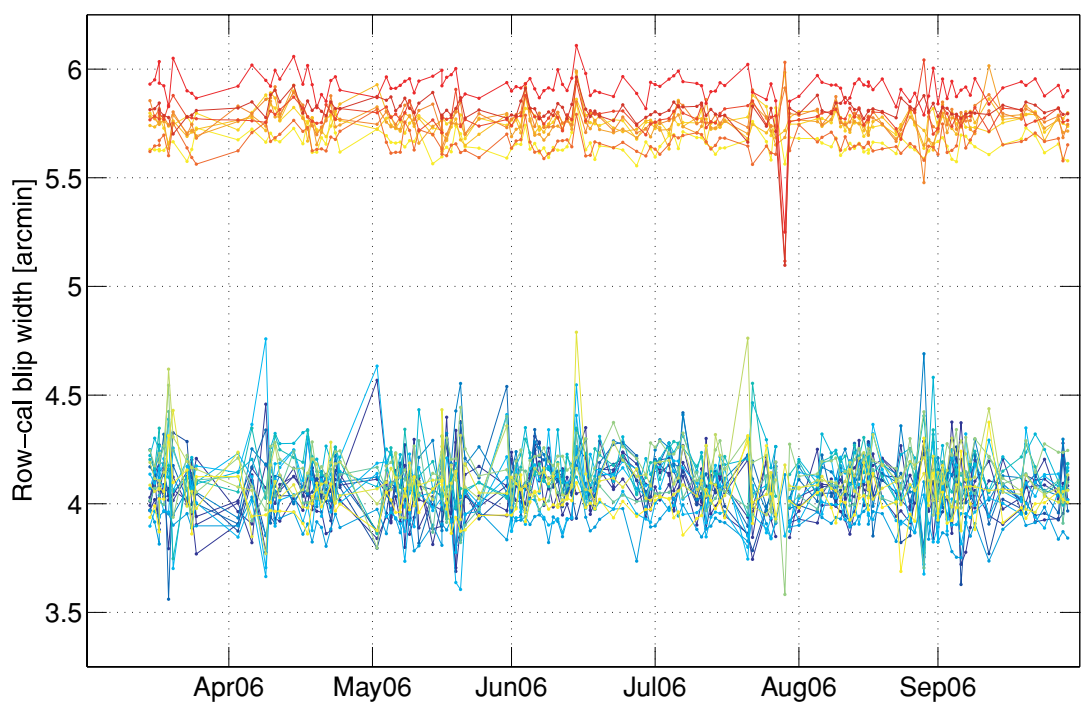

Figure 20. The beam width of each feed horn, as measured from daily "row-cal" scans over RCW38. Azimuth scans are made at seven elevation offsets from the source, corresponding to the center of each row of feed horns in the focal plane. Gaussian profiles are fitted to the blip in the time stream corresponding to the source. Because full raster maps are not made, pointing wander results in errors in the derived beam widths. The larger scatter at $150 \mathrm{GHz}$ results from the higher atmospheric noise present in this band. There is no evidence for long-term drift in the beam width over the course of a season.

(A color version of this figure is available in the online journal.)
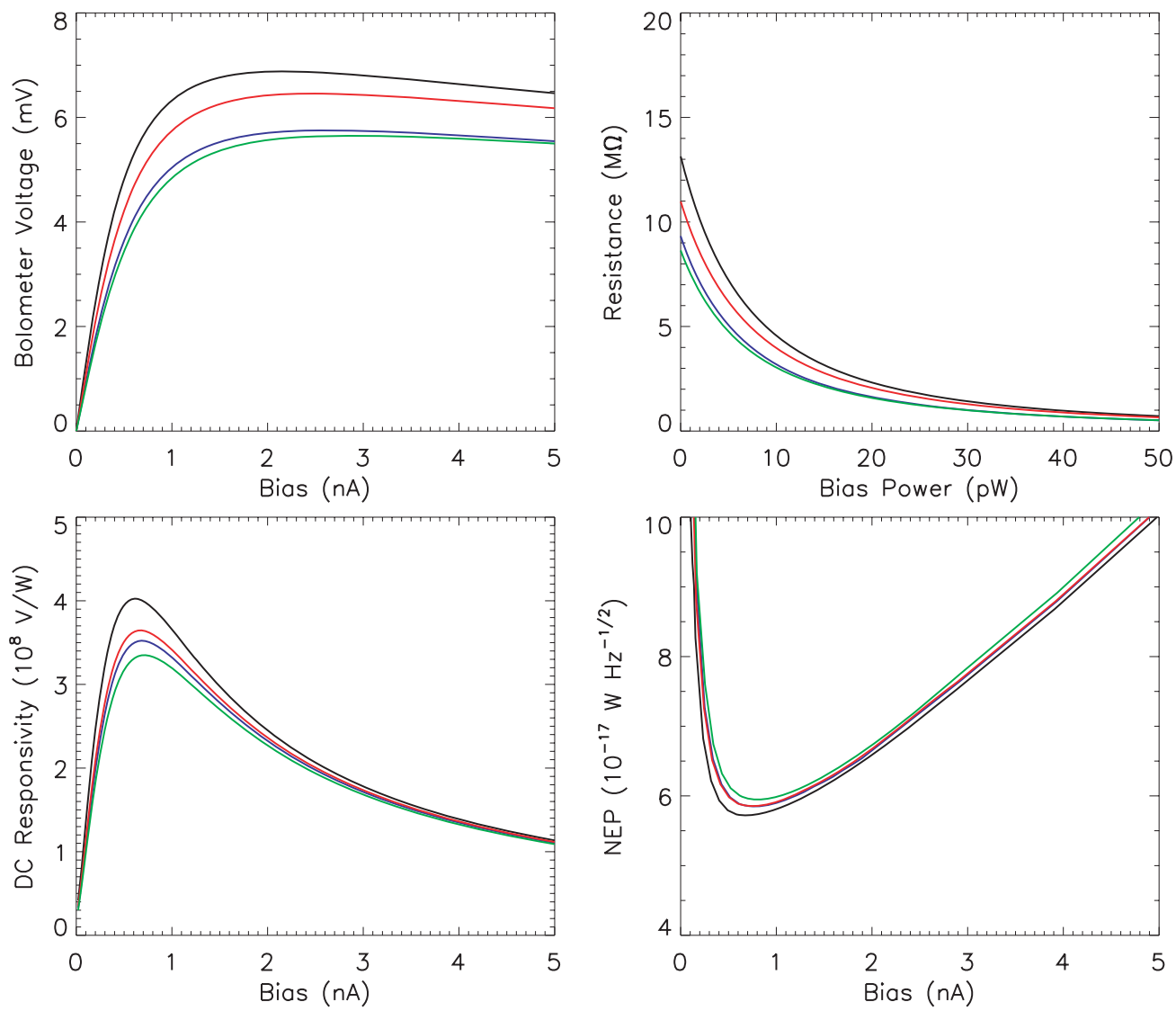

Figure 21. Load curves for four $150 \mathrm{GHz}$ QUaD detectors taken on 2005 May 31 plotted in different units. For low bias current, bolometers obey Ohm's law, but increased bias results in self-heating that decreases resistance. These load curves were taken as part of a routine calibration set during CMB observations at an elevation of $48^{\circ}$ and a baseplate temperature of $255 \mathrm{mK}$. During observations, the detector bias current is set at $1.2 \mathrm{nA}$.

(A color version of this figure is available in the online journal.)

points are $f_{3 \mathrm{~dB}}=20 \mathrm{~Hz}$ for $H_{1}$ and $30 \mathrm{~Hz}$ for $H_{2}$. This transfer function was verified by laboratory measurements and deviations from the model were found to be negligible over the frequency range of interest for $\mathrm{CMB}$ measurements $(0.1-2 \mathrm{~Hz})$.
The bolometer response dominates the electronics response in the science band and accurate measurements of $H_{b}$ are essential in order to correctly measure the CMB power spectrum. The response function of an ideal bolometer is $S(\omega)=S_{\mathrm{DC}} /(1+$ 

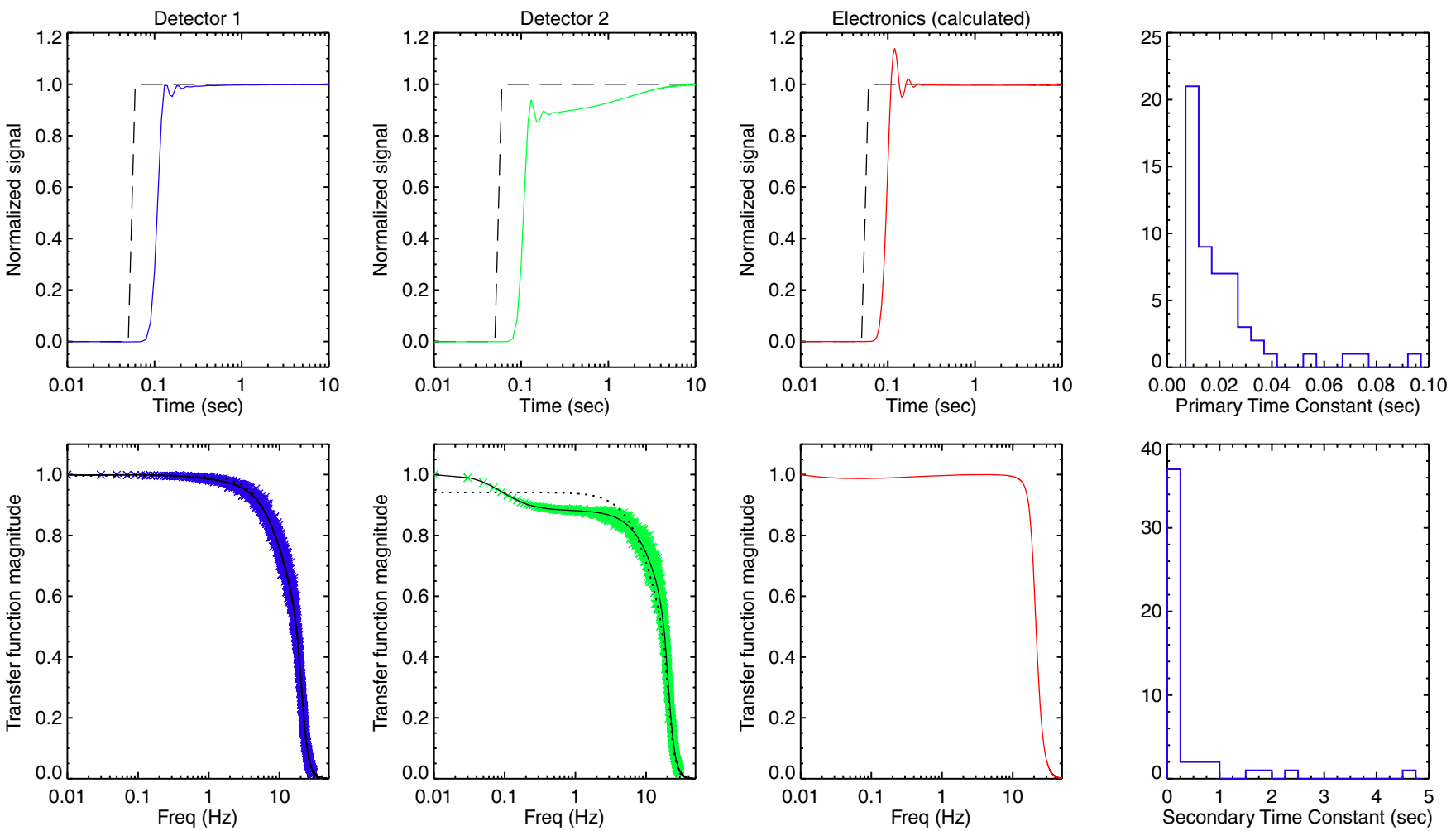

Figure 22. A Gunn diode source was used to supply a square wave signal with fast edge transitions to the focal plane in order to measure the detector time constants. Left: the Gunn diode reference signal (black dashed) and the measured step response of two detectors (blue and green). The initial $\sim 150 \mathrm{~ms}$ is dominated by the electronics transfer function which introduces the overshoot and ringing (red). The transfer function for the two detectors, derived from Fourier transforming the time-domain data, is shown below the time ordered data. Fits to a single time constant model (dotted) and a dual time constant model (solid) are shown. The two right-hand panels show the distribution of both the fast (upper right) and slow (lower right) time constants for all of the QUaD detectors.

(A color version of this figure is available in the online journal.)

$i \omega \tau$ ) where $S$ is the detector responsivity (Volts/Watt), and the time constant $\tau=C / G$ where $C$ is the heat capacity of the absorber and $G$ is the thermal conductivity between the sensor and the bath. Values for $\tau$ of order $30 \mathrm{~ms}$ are typical of QUaD detectors although there is substantial device-to-device variation. Both $C$ and $G$ are temperature dependent, so it is essential to measure time constants with similar optical loading and electrical bias power as during observation.

Two methods were used to determine the time constants for the QUaD detectors. The first method involved scanning the telescope back and forth in the azimuth over RCW38 and recording the shift in the apparent position of the source for the forward and backward scan directions. The second method involved a special test run during the Austral summer. The telescope was illuminated with a Gunn diode RF source that was chopped with a slow (100 s) square wave. This measures the system step response from which the transfer function can be derived (Figure 22). Care was taken to ensure that the Gunn setup did not substantially increase the optical loading on the detectors. The results of the two methods are in excellent agreement with each other; however, the Gunn measurements offered a much larger signal-to-noise ratio, and these are the values used in the Data Paper.

It was found that for about half the detectors the single time constant model is a poor fit to the true response. The addition of a second time constant term, so that the overall detector transfer function is modeled as

$$
H_{b}(\omega)=\frac{1-\alpha}{1+i \omega \tau_{1}}+\frac{\alpha}{1+i \omega \tau_{2}}
$$

where $\alpha \leqslant 0.5$, was found to accurately model the detector response in all but two cases - these detectors were not used. In some cases, the second time constant is found to be extremely long $\left(\tau_{2}>1 \mathrm{~s}\right)$, but is still very well fitted by the double time constant model. These detectors are used in the science analysis. The physical origin of the second time constant is believed to be debris on the PSB which is in weak thermal contact with the absorbing grid. Figure 22 shows the transfer functions for two detectors, and the distribution of time constants for all the detectors used in the season $2 / 3$ analysis.

\subsection{Polarization Angles and Cross-Polar Leakage}

The voltage response of a single PSB to arbitrarily polarized incident radiation is given by

$$
v=s\left[\mathcal{I}+\frac{1-\epsilon}{1+\epsilon}(\mathcal{Q} \cos 2 \theta+\mathcal{U} \sin 2 \theta)\right],
$$

where $\theta$ gives the orientation angle of the PSB, $\epsilon$ gives the cross-polar leakage, and $s$ is a calibration constant that depends on detector responsivity, optical throughput $(A \Omega)$, optical efficiency, bandwidth, and readout electronics gain (Jones et al. 2003). From Equation (5), leakage can be seen to result in a loss of optical efficiency to polarized radiation. This has the effect of reducing sensitivity (Section 10) but not of mixing Stokes parameters. The measurement of $\epsilon$ and $\theta$ is described in this section. The determination of the calibration constant, $s$, is described in Section 9.

Because there are no bright, well calibrated polarized astronomical sources visible at these wavelengths, we characterized 

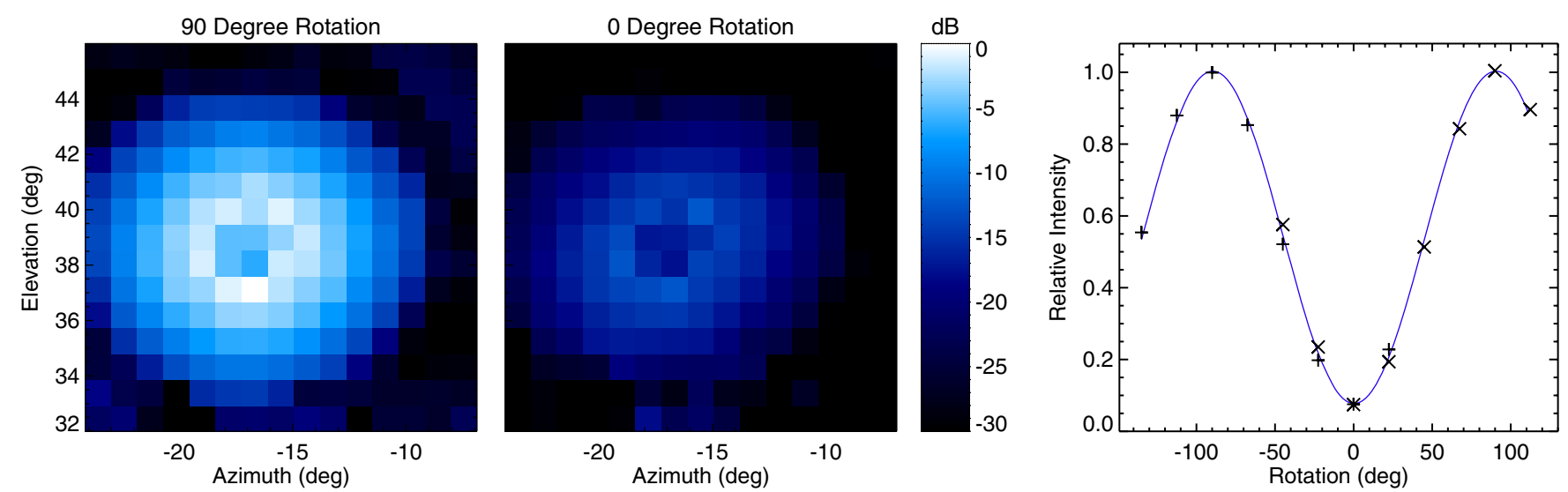

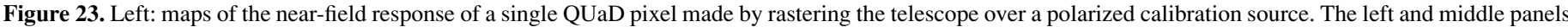

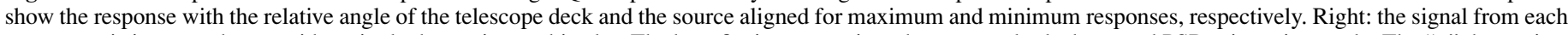

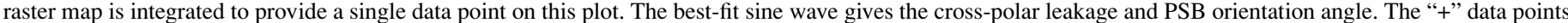

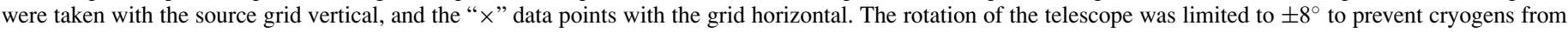
spilling.

(A color version of this figure is available in the online journal.)

the polarized response of the instrument by observing a nearfield, polarized source mounted on a tower located outside the ground shield. The source consisted of a rotating chopper wheel viewed through a circular aperture and a linear polarizing grid. ${ }^{34}$ The chopper wheel alternated between ambient temperature Eccosorb and reflective aluminum aimed at cold sky with a modulation rate of $5 \mathrm{~Hz}$. A phase-synchronous reference signal was digitized on one of the spare analog input channels and was used to demodulate the bolometer voltages in post-processing.

A series of $15^{\circ} \times 15^{\circ}$ raster maps of the calibration source were recorded, rotating the receiver about the optical axis in steps of 22.5 between each map. After eight maps, the source grid was rotated by $90^{\circ}$ and the entire process was repeated. Each raster map required approximately 45 minutes of observation. Two complete polarization calibration runs were performed two months apart, during the second season of observation. For the first run, a source aperture made from aluminum was used. For the second run, an Eccosorb aperture was used. Results from the two runs are similar, with slightly lower values of $\epsilon$ obtained on the second run due to less depolarizing effects from the source aperture.

The signal from each raster map is integrated to provide a single data point on a plot of measured signal versus relative grid/PSB orientation angle (Figure 23). These data points are then fitted to a sine wave. The cross-polar leakage, $\epsilon$, is given by the difference between the sine wave minimum and the $x$ axis. The absolute orientation angle of the PSB is given by the phase angle of the sine wave.

Figure 24 shows the results of these two polarization calibration runs. We find that the average cross-polar leakage of all the channels that are used in the season $2 / 3$ analysis is 0.08 . In order to check for systematics in the measurement of $\epsilon$ and $\theta$, the results from the two calibration runs can be compared, and additionally each calibration run can be split into two halves, since half of the data points were taken with the source grid aligned horizontally and half vertically. By comparing the results obtained from independently analyzing the "grid horizontal" and "grid vertical" data subsets, and the results from the two sepa-

\footnotetext{
34 The polarizing grid, manufactured in Cardiff, is an array of copper traces at a $10 \mu \mathrm{m}$ pitch supported on a thin polypropylene sheet. At $150 \mathrm{GHz}$, it was measured to have a polarization efficiency of greater than $99 \%$.
}

rate calibration runs, an uncertainty on the mean value of $\epsilon$ of $\sim \pm 0.02$ is obtained.

The measurements of cross-polar leakage are consistent with laboratory measurements of individual pixels made using a small test bed cryostat prior to integration of the QUaD receiver. The optical system of the test cryostat is simpler than in the full receiver, containing only filters and a vacuum window and no lenses. Multiple feeds and detectors at each frequency were characterized in the test bed. This suggests that the lenses and the telescope are not significant sources of cross-polar leakage.

The rms scatter of the PSB angles about their nominal orientation was found to be 1.2 , and the deviation from orthogonality within a pair was similarly of order $1^{\circ}$. Several mechanisms contribute to the errors in PSB orientation including machining tolerances of the focal plane plate and PSB modules, transmission through the cryostat optical chain, and the alignment of the absorber within the PSB module. Simulations show that random offsets in the PSB angles at this level average down and have no effect on the resulting CMB polarization power spectra.

A larger $\left(\sim 2^{\circ}\right)$ systematic uncertainty in the absolute position of the PSB orientation angles results from differences obtained in the two calibration runs and from separate fits to the source grid horizontal and source grid vertical data subsets. Systematic rotation of the detectors is more problematic than random scatter since it does not average down when detector signals are combined. Misalignment produces leakage of the dominant CMB E-mode power into the much weaker B mode signal. However, simulations show that at QUaD's sensitivity a rotation of order $10^{\circ}$ - five times larger than the actual uncertainty-is necessary to produce a detectable amount of spurious BB signal.

\subsection{Optical Loading}

Optical loading on the detectors arises from thermal emission in the instrument, from atmospheric emission and from astronomical sources, including the CMB. The total loading is

$$
T_{\text {load }}(\theta)=T_{\text {receiver }}+T_{\text {telescope }}+(1-\mathcal{T}) T_{\text {atm }}+\mathcal{T} T_{\mathrm{CMB}},
$$

where $T_{\text {atm }}$ and $T_{\mathrm{CMB}}$ are the Raleigh-Jeans temperatures of the atmosphere and the $\mathrm{CMB}$. The loading per detector averages $\sim 30 \mathrm{~K}$ (Table 3 ). 

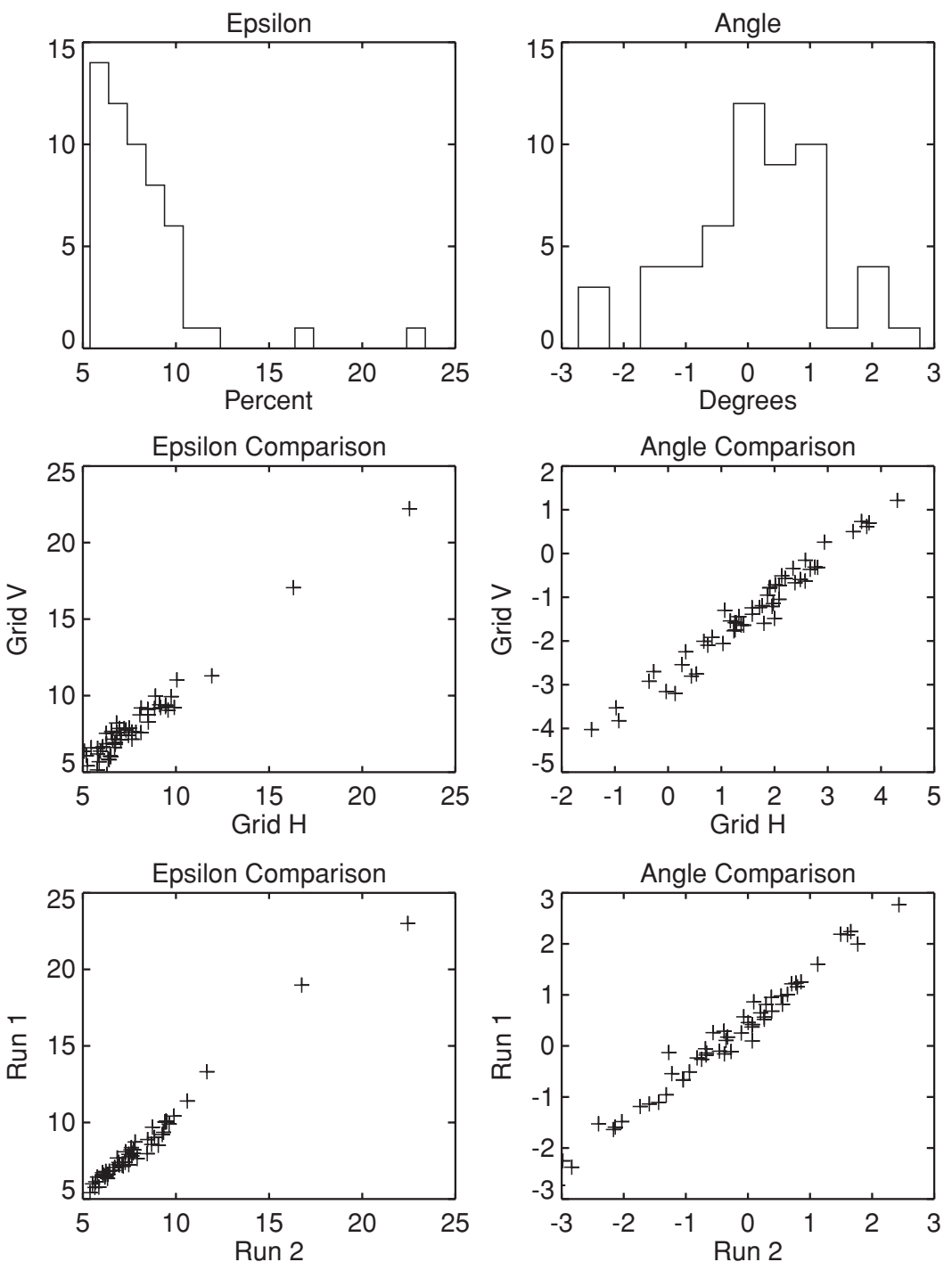

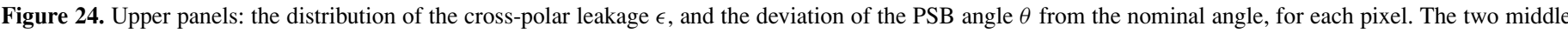

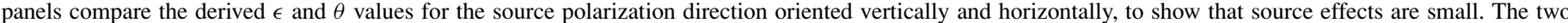

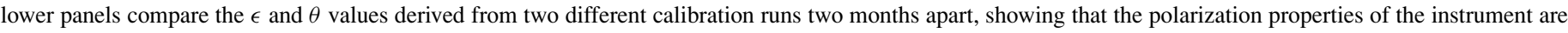
stable with time.

Excess loading reduces sensitivity by increasing the photon noise contribution and by warming the detectors, which reduces responsivity (Section 10). The QUaD receiver was designed to minimize internal loading. All transmissive components including the window, lenses, and filters are AR coated with thin layers of intermediate index material. Also a blackened cold stop located on the $4 \mathrm{~K}$ stage intercepts the sidelobes from the feed horns.

For QUaD, the atmospheric transmission is measured daily with a procedure known as a skydip, during which the detector voltages are recorded as the telescope is tipped in elevation from zenith to $45^{\circ}$. The atmospheric transmission depends on the zenith angle as $\mathcal{T}=e^{-\tau / \cos \theta}$ where the optical depth, $\tau$, depends on the frequency band of observation. The combination of the excellent atmospheric conditions at the South Pole, with $\tau$ at $150 \mathrm{GHz}$ less than $\sim 0.04$, and the limited elevation range accessible to QUaD means that $\mathcal{T} \approx 1-\tau / \cos \theta$ and the total loading temperature is given by

$$
T_{\mathrm{load}}(\theta) \approx T_{0}+\frac{\tau T_{\mathrm{atm}}}{\cos \theta}
$$

Consequently it is not possible to separately fit for $\tau$ and $T_{\text {atm }}$ from QUaD skydip data alone. The degeneracy was broken by using data from the AST/RO $350 \mu \mathrm{m}$ tipper which is located on an adjacent building and which measures $T_{\text {atm }}$ (and $\tau$ at $350 \mu \mathrm{m}$ ) several times per hour. By using the tipper measurement of $T_{\text {atm }}$, the value of $\tau$ at the QUaD observing frequencies can be derived. ${ }^{35}$ During routine observing, QUaD performs one skydip per day.

Figure 25 shows the measured $\tau$ values for the 2006 season. It can be seen that the derived optical depth is larger at $100 \mathrm{GHz}$ than at $150 \mathrm{GHz}$, as expected from the atmospheric transmission model in Figure 7. The optical depth is, however, more variable at $150 \mathrm{GHz}$ than at $100 \mathrm{GHz}$ because the former is more sensitive to the water vapor content. A comparison of the $350 \mu \mathrm{m}$ tipper data and the $150 \mathrm{GHz}$ optical depth derived from QUaD skydips gives a best-fit linear relationship of

$$
\tau_{150}=0.020+0.0127 \times \tau_{350 \mu \mathrm{m}}
$$

and shows that the two are strongly correlated.

\footnotetext{
35 The atmospheric temperature, $T_{\mathrm{atm}}$, is a weighted, line-of-sight-averaged temperature and is not, in general, the same as the surface air temperature.
} 

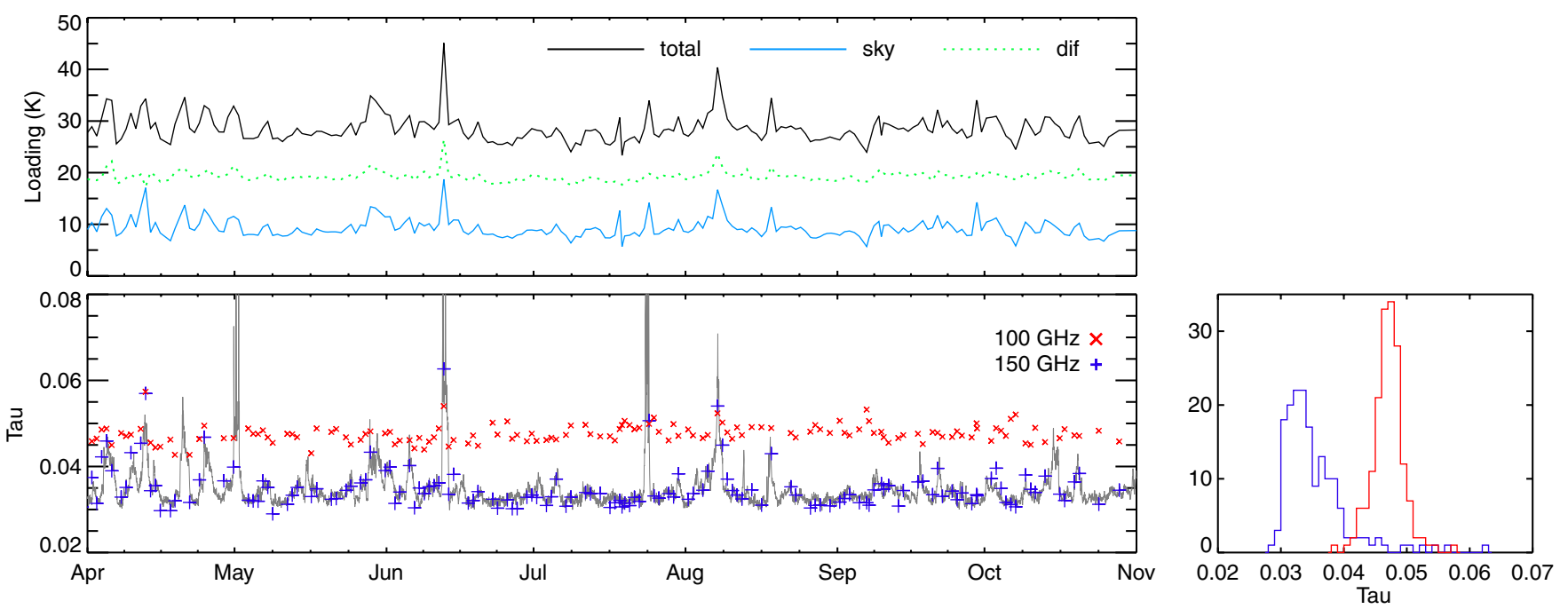

Figure 25. Top: average Raleigh-Jeans optical loading temperature for the $150 \mathrm{GHz}$ channels, measured during the 2006 observing season. The total loading (black) is derived from daily load curves and the atmospheric loading (light blue) is derived from daily skydip measurements. The difference (dotted green) remains flat over the season, indicating that the loading is not increasing due to, for example, snow accumulation on the foam cone. Bottom: atmospheric optical depth $(\tau)$, as measured by daily QUaD skydips for both observing bands. Data from the AST/RO $350 \mu \mathrm{m}$ tipper (located in a neighboring building) is shown in gray. The gray line shows an extrapolation of the $350 \mu \mathrm{m}$ optical depth from the tipper data to $150 \mathrm{GHz}$ using the relation in the text. There is excellent agreement between the two. Right: histogram of the 100 (red) and $150 \mathrm{GHz}$ (blue) tau measurements.

(A color version of this figure is available in the online journal.)

Table 3

Loading, Noise Budget, and Sensitivity

\begin{tabular}{lcc}
\hline \hline Frequency Band (GHz) & 100 & 150 \\
\hline Loading (Temperatures are Raleigh-Jeans) & & \\
Total loading per detector (pW) & 2.8 & 5.1 \\
Total loading per detector $(\mathrm{K})$ & 27 & 26 \\
Atmospheric loading $(\mathrm{K})$ & 11 & 9 \\
Telescope loading $(\mathrm{K})$ & 8 & 8 \\
CMB loading $(\mathrm{K})$ & 1.1 & 0.6 \\
Bolometer parameters & & \\
$R_{0}\left(10^{6} \Omega\right)$ & 94.1 & 94.8 \\
$\Delta(\mathrm{K})$ & 42.0 & 42.1 \\
$T_{\text {Bolo }}(\mathrm{K})$ & 0.356 & 0.357 \\
$G\left(\mathrm{pW} \mathrm{K}{ }^{-1}\right)$ & 147 & 156 \\
Responsivity $\left(10^{8} \mathrm{~V} \mathrm{~W}^{-1}\right)$ & 3.6 & 3.1 \\
Noise Equivalent Power $\left(10^{-17} \mathrm{~W} / \mathrm{Hz}^{1 / 2}\right)$ & & \\
NEP amplifier & 2.5 & 3.0 \\
NEP Johnson & 1.4 & 1.7 \\
NEP Phonon & 2.5 & 2.7 \\
NEP photon shot & 1.9 & 3.2 \\
NEP photon Bose & 1.7 & 2.5 \\
Total NEP without Bose & 4.3 & 5.4 \\
Total NEP with Bose & 5.6 & 5.9 \\
NEP achieved & 4.7 & 5.8 \\
Sensitivity & & \\
Calibration $\left(\mathrm{K} \mathrm{V}^{-1}\right)$ & 0.55 & 0.47 \\
NET per feed $\left(\mu \mathrm{K} \mathrm{s}^{-1 / 2}\right)$ & 440 & 390 \\
NEQ per feed $\left(\mu \mathrm{K} \mathrm{s}^{-1 / 2}\right)$ & 510 & 450 \\
\hline
\end{tabular}

Note. Values are for a good observing day at the field elevation of $\sim 50^{\circ}$. See Section 10.

\section{REJECTION OF SPURIOUS SIGNALS}

All bolometric detector systems are susceptible to contamination from a variety of effects including stray light, crosstalk, radio frequency interference (RFI), and microphonic pickup. Although the differential nature of polarization measurements provides an extra level of rejection compared to total-power measurements, careful design is still required. To monitor any residual effect, the focal plane includes several dark bolometers and fixed resistors.

\subsection{Stray Light}

The QUaD bolometer modules are not light tight, so a $250 \mathrm{mK}$ shield is mounted beneath the focal plane bowl to prevent stray light from entering through the modules themselves. All joints in the shield are stepped to provide a convoluted path for light, and the inside is blackened with carbon-loaded Stycast 2850FT. The volume enclosed by the focal plane bowl and the shield is vented through small-diameter, teflon tubes embedded in the absorber. The long tubes are bent and glued to the inside of the shield to remove any light path while still providing an exit for gas. Load curves of the dark bolometers show negligible stray light inside the focal plane enclosure. Correlations of the output signals from the dark bolometers with the signal from light channels made while the receiver observes a modulated optical load show that cross-talk (both optical and electrical) is less than $1 \%$.

\subsection{Electrical Pickup and Radio Frequency Interference}

QUaD is well protected from pickup in the warm readout electronics and cabling. The electronics boxes mount onto RFtight interface boxes which themselves attach directly to the cryostat bottom flange and are sealed with the RF braid. All signals entering or exiting the interface boxes pass through filtered D-Sub connectors (Section 6). The signals are again filtered at the $4 \mathrm{~K}$ bulkhead, this time with filtered micro-D connectors. The signal paths for the two halves of each PSB module are kept as similar as possible, so that any electrical pickup will affect each in a similar manner and will be attenuated by differencing.

RFI in the low-GHz range is particularly insidious as it easily enters the cryostat through the window, where it is picked up by the signal wiring and then coupled to the detectors. Any RF 


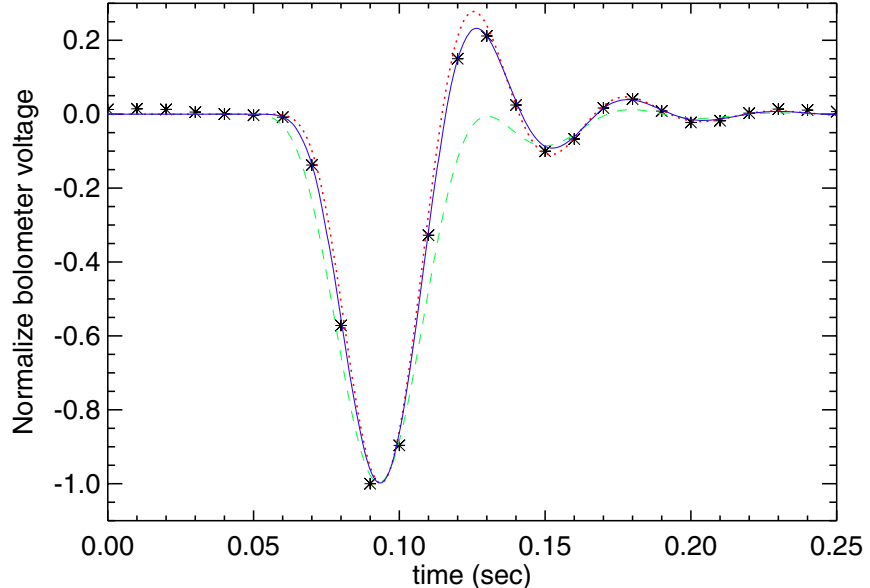

Figure 26. The response of a channel to a cosmic ray hit. The crosses show the measured data points. A good fit is given by the transfer function described in Section 7.5 with the detector time constant left as a free parameter (solid blue curve). In this case, the best-fit detector time constant is $6 \mathrm{~ms}$, much shorter than the optically measured value of $19 \mathrm{~ms}$ (dashed green). The electronics-only impulse response is given by the dotted red curve.

(A color version of this figure is available in the online journal.)

power picked up in this way will heat the thermistors, causing a decrease in voltage that can be confused with true signal. Fortunately, the RF environment at the remote South Pole site is both exceptionally clean and tightly controlled, especially during the Austral winter. All communication and weathermonitoring transmissions are in the low- $\mathrm{MHz}$ region of the spectrum and are thus blocked by the size of QUaD's optical snout. During three years of observations, no contaminating transmissions were detected by the instrument during the winter observing season.

\subsection{Microphonic Pickup}

Mechanical vibrations in the high-impedance signal wiring between the detectors and the JFET buffers can cause spurious signals - an effect known as microphonic pickup. To mitigate this effect, the JFET buffer amplifiers are placed as close as possible to the detectors, reducing the length of the susceptible wiring, and all the high-impedance wiring is carefully tied down along its entire path to limit vibration. Additionally, the QUaD mount has extremely smooth tracking; accelerometer measurements showed the vibration level to be under $0.5 \times$ $10^{-4} \mathrm{~g} \mathrm{~Hz}^{-\frac{1}{2}}$ within the range of $40-200 \mathrm{~Hz}$ during typical azimuth scans. Finally, the phase compensation in the lockin amplifiers is frequently adjusted which minimizes sensitivity to microphonically induced phase shifts.

Although these measures significantly reduce the amount of microphonic pickup, there remain some broad resonant features in the output signals. However, the adjustable bias frequency allows operation in a clean region so that the signal frequencies remain uncontaminated. With the bias frequency properly adjusted, the receiver shows no increase in white noise level or the $1 / f$-knee frequencies, even during telescope motion.

\subsection{Cosmic Rays}

Cosmic ray hits cause a short glitch in the data stream of the affected channel (Woodcraft et al. 2003). See Figure 27 for a typical cosmic ray event. The PSBs used in QUaD have a small cross section to cosmic rays, so these events occur infrequently. During observation at the South Pole, QUaD experienced approximately 0.5 cosmic ray events per channel per hour. These events are tagged in the low-level data reduction, and contaminated regions are excluded from further analysis. This results in a loss of data of much less than $1 \%$.

Cosmic ray impacts closely approximate delta function power inputs, providing a measure of the system impulse response function. These events are typically well fitted using the transfer function described in Section 7.5, with the detector time constant left as a free parameter. The best-fit detector time constants are shorter than those obtained from optical measurements (on average, by a factor of 3). They vary for different events on the same device and show little correlation with the optically measured time constants. These effects are due to poor thermal conductance across the PSB absorber combined with the random impact location of each event. Cosmic ray glitches are thus not useful for measuring the detector time constants, but they do provide a monitor of the electronics impulse response.

\subsection{Sidelobe Pickup}

Figure 27 shows the location of the telescope sidelobes. The inner circular sidelobe arises from reflection off the cryostat collar baffle (Section 3). The radial sidelobe, caused by the truncation of the beam inside the cryostat, is present in the outer ring of $150 \mathrm{GHz}$ channels. Finally, all channels exhibit an outer circular sidelobe at approximately $100^{\circ}$ from the optical axis caused by reflection off the foam cone. These sidelobes have been extensively studied with a series of special mapping runs, as described in this section.

An optics model was used to estimate the amplitude of these sidelobes. Of the power leaving a feed horn, approximately $5 \%$ passes through the hole in the secondary mirror. Another $10 \%$ is reflected from the cryostat baffle to the inner circular sidelobe. Combined, these two terms represent the loss of efficiency that results from blockage by the on-axis secondary mirror. A further $\sim 2 \%$ of the power is lost due to reflection off the foam cone. Half of this power forms the narrow, $100^{\circ}$ sidelobe, while the rest scatters off the primary resulting in a wide, diffuse sidelobe at $\sim 30^{\circ}$ off the boresight.

The inner circular sidelobe and the radial lobe were studied with a series of special mapping runs using a chopped Gunn diode mounted on the top of the ground shield as a source. The results of these runs are shown in Figure 28. After these tests, it was decided to replace the original first season parabolic baffle with a flat, reflecting baffle. This moved the sidelobe from $\sim 25^{\circ}$ off the optical axis to $\sim 10^{\circ}$ for seasons 2 and 3 , where it would be less likely to intercept a contaminating source. The total power in this sidelobe remains the same.

As a further test during the summer of 2006, the annular cryostat baffle was coated in absorbing material, and a cylindrical absorbing baffle was placed above the cryostat snout. Tests with the Gunn source indicated that these baffles eliminated the inner circular sidelobe and the radial lobe. However, the increase in the average detector loading from these baffles resulted in an unacceptable loss of sensitivity and they were removed before beginning CMB observations. With just the absorbing collar baffle, an average of $10 \mathrm{~K}$ increased loading was seen at $150 \mathrm{GHz}$ versus a $20 \mathrm{~K}$ increase with both baffles. There were channelto-channel variations of order a factor of 2 . This indicates that the inner circular sidelobe and radial sidelobe are of similar strength. These loading increases are slightly less than expected from the optics model.

The sidelobes can introduce spurious signals into the data via pickup from the ground as the telescope scans or if they 


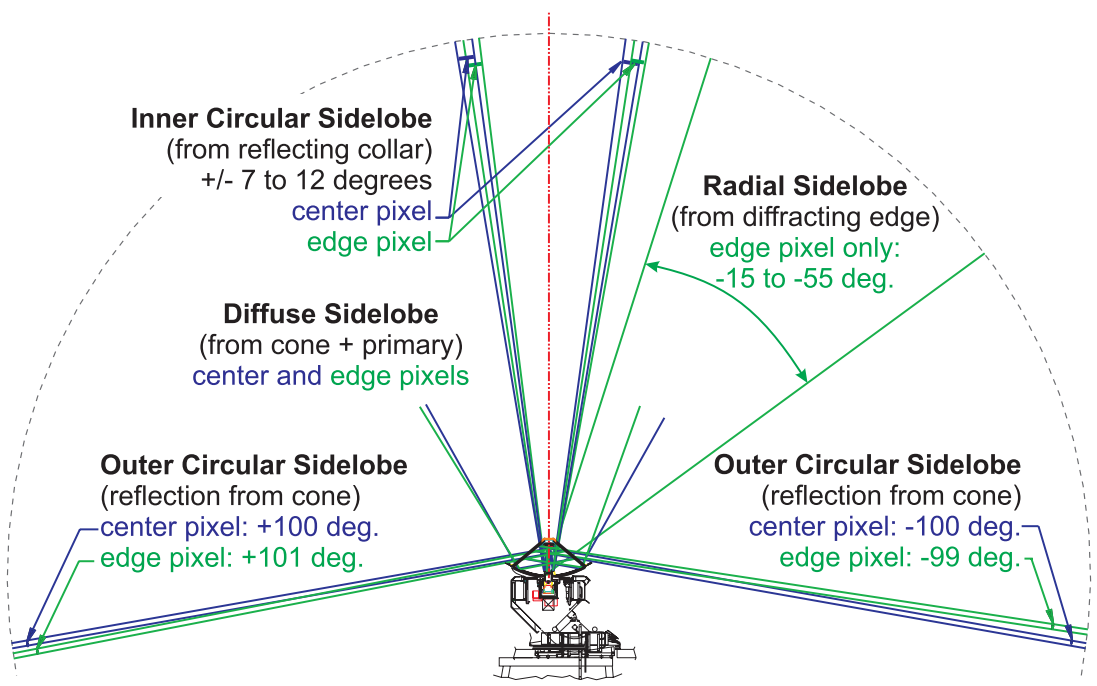

Figure 27. Schematic showing the location and origin of the telescope sidelobes.

(A color version of this figure is available in the online journal.)
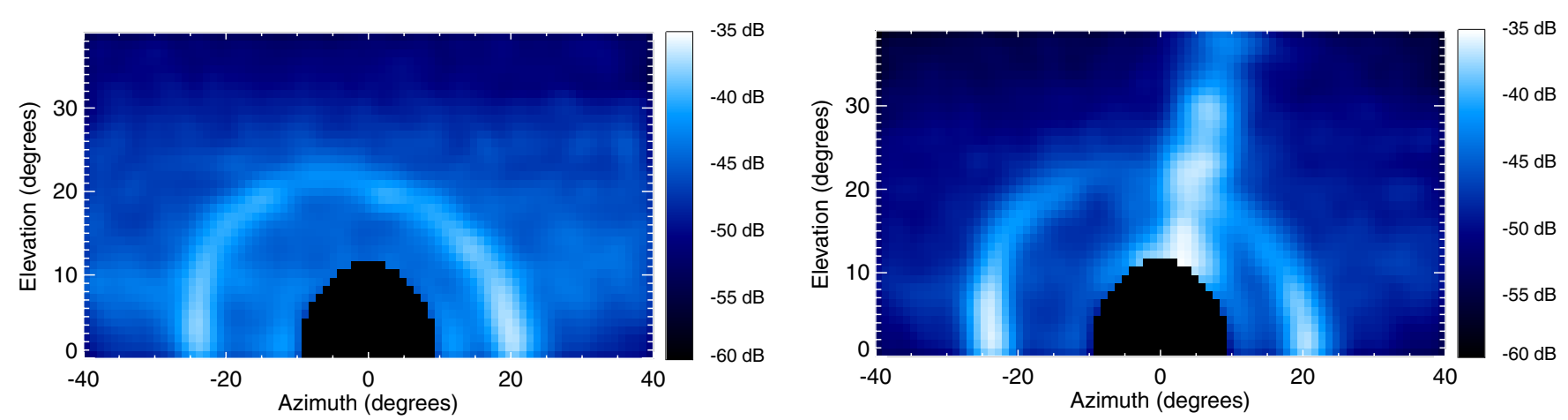

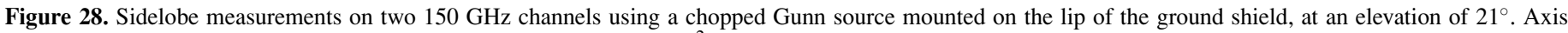

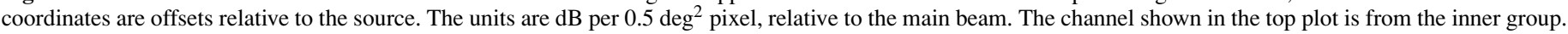

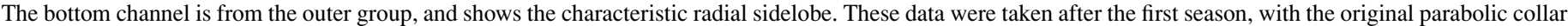
baffle.

(A color version of this figure is available in the online journal.)

intercept a bright astronomical source. Despite the reflective ground shield surrounding the telescope, low-level ground pickup is seen in the raw data. The ground signal appears at low frequencies and is very effectively suppressed by highpass filtering of each scan. Furthermore, a field differencing observation strategy was employed for all CMB observations. Two neighboring CMB fields, separated by exactly 30 minutes in right ascension, were observed. The "lead" field was observed first, and the "trail" field was observed precisely 30 sidereal minutes later, with the telescope repeating an identical scan path with respect to the ground. The difference field (lead-trail) is free of any ground pickup that is stable on time scales of 30 minutes or longer.

Because of the low level of the sidelobes, only bright astronomical sources can create contamination. The Sun and planets are always below the ground shield during $\mathrm{CMB}$ observations. Pickup from the galactic plane was simulated and found to contribute at a level well below the sensitivity of QUaD. This leaves the Moon as the most problematic source. The geometry of the site and field location confines the Moon to a "space angle" range of $\sim 75^{\circ}-115^{\circ}$ measured with respect to the telescope boresight. Therefore, Moon contamination only enters the data via the $100^{\circ}$ sidelobe.
The Moon, if it is appropriately positioned, leaves a characteristic stripe in the maps, at an angle consistent with its rate of movement across the sky (Figure 29, left). A model was developed that accurately predicts when Moon contamination will occur based on the relative position of the Moon to the telescope (Figure 29, right). This model was used to cut any days with possible contamination, even if no visible evidence was seen in the maps.

Although the foam cone was designed to have a nominal opening angle of $100^{\circ}$, in practice this can vary due to thermal expansion/contraction of the cone, inhomogeneities in the adhesive, small tilts of the cone with respect to the boresight, and to a lesser extent mechanical loading. These effects cause the sidelobe space angle to fluctuate slightly as a function of time and of telescope deck rotation angle. We found that pickup from the moon in the CMB data occurs over the range $96.5<$ space angle $<101^{\circ}$, and this forms the basis for our very conservative Moon cuts as discussed in the Data Paper.

The $100^{\circ}$ sidelobe was studied in Austral summer 2007 with a series of runs during which it was deliberately scanned across the sun while the telescope rotated through the full 360 degrees about the boresight (deck rotation). These were intended to precisely map the variation of space angle with deck, but due 

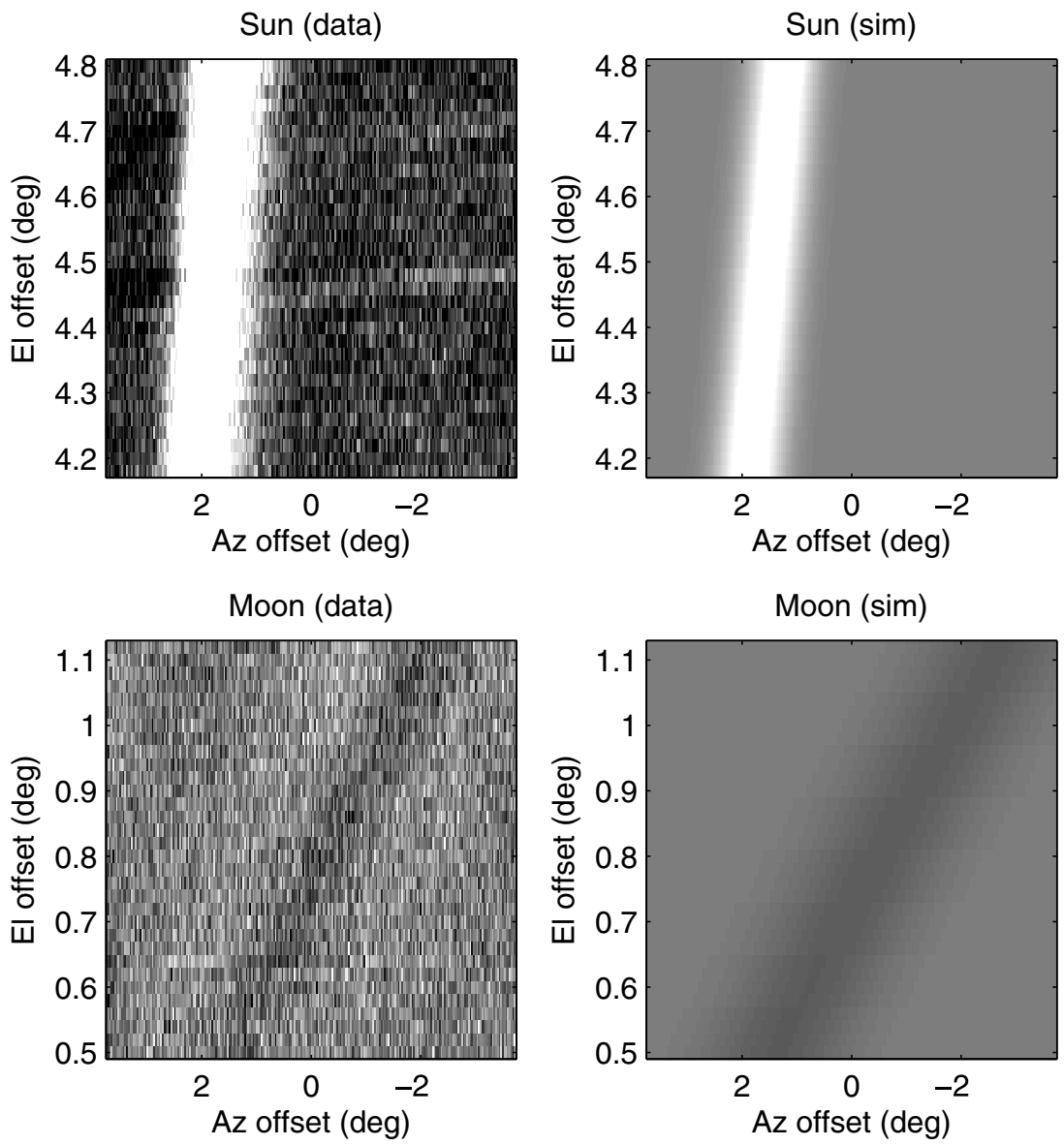

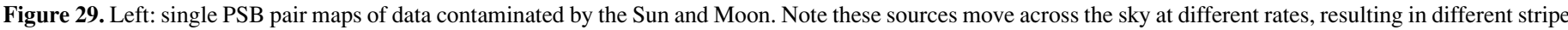

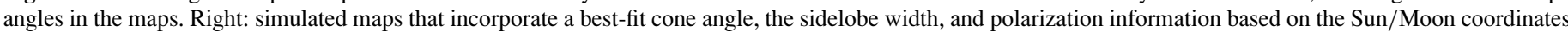
during the same day as the real data. The cone opening angle (allowed to vary from $96^{\circ}$ to $101^{\circ}$ ) is the only free parameter of this model.

to differing weather conditions in summer and winter, we could not use these results to provide tighter cuts on the CMB data. These runs did, however, confirm that the sidelobe is extremely narrow $(\mathrm{FWHM} \sim 0.22)$.

Since it is the result of reflection off a dielectric surface, the sidelobe is highly polarized. Laboratory tests using a foam cone structure identical to that on the telescope measured the fractional polarization to be $50 \%$ at $150 \mathrm{GHz}$, with a reflection coefficient of approximately $2 \%$. The amplitude of the electric field of a reflected ray in the radial direction to the cone axis of symmetry is suppressed relative to that in the tangential direction. A PSB with its sensitivity axis aligned with the cone tangent will therefore measure a larger signal than a perpendicular PSB, resulting in a false polarized signal in the differenced data. We can predict the sign of the polarized signal if we know the PSB pair orientations, and the angle at which a bright source appears in the frame of the focal plane (Figure 30). The amplitude of the Moon contamination signal $(\sim 3 \mathrm{mK})$ is consistent with these laboratory foam cone transmission measurements, assuming a Moon brightness temperature of order $300 \mathrm{~K}$.

\section{CALIBRATION}

The QUaD calibration process comprises frequent relative calibrations, and an absolute calibration of the final maps based on comparison with CMB temperature measurements from other experiments. The primary method of channel-to-channel relative gain calibration is to nod the telescope in elevation by $1^{\circ}$ (known as an "el-nod"). The resulting change in air mass generates an approximately linear signal of order a Volt in the amplified time stream of each detector. The measured signal is used to normalize the gain of each detector to the mean gain of all detectors in a band, and includes a correction for the elevation offset of the feeds in the focal plane at the current telescope rotation angle. Figure 31 shows an example of an el-nod and the measured distribution of relative detector gains. During routine CMB observations, el-nods are performed during a "cal set" that precedes each scan set, approximately every 30 minutes.

Each cal set also includes a run of the calibration source, which is located above the secondary mirror, inside the foam cone (Figure 32). When in use, a flip mirror reflects the portion of the beam passing through the secondary hole onto a rotating polarizing grid in front of an ambient-temperature blackbody target, introducing a sinusoidally modulated signal into the detector time streams. To avoid running wires along the foam cone which could introduce spurious polarized signals, the calibration source is battery powered and controlled via an infrared link. The calibration source does not uniformly illuminate the focal plane, and hence cannot be used to correct for relative channel-to-channel gain variation-this is done with the el-nods. Instead, the calibration source is used to correct for drifts in the mean receiver gain over time, an effect the el-nods alone cannot correct since gain drifts and changes in atmospheric loading both affect the el-nod volts/airmass in the same way. 
PSB pair orientation; $\mathrm{dk}=70^{\circ}$
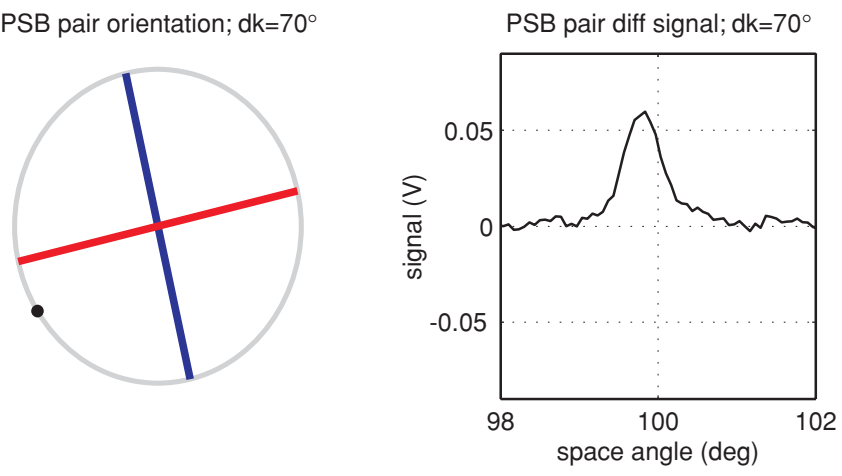

PSB pair orientation; $\mathrm{dk}=160^{\circ}$

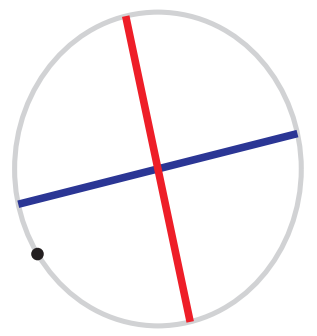

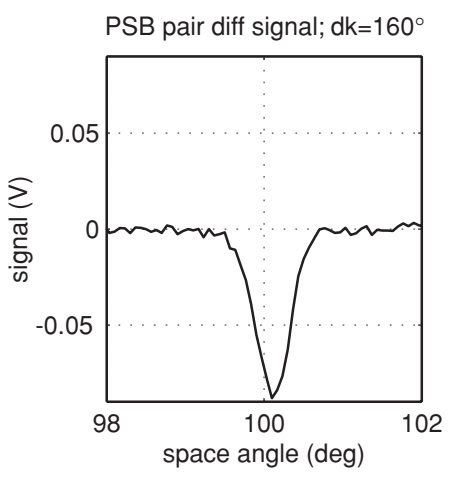

Figure 30. PSB pair orientation with respect to the Sun position in the frame of the focal plane, for two telescope deck rotation angles separated by $90^{\circ}$. As expected, the pair differenced (A-B) signal is positive when detector A (blue) is perpendicular to the radial direction to the source. Likewise, with the Sun in a very similar position, the pair rotated by $90^{\circ}$ produces a negative pair difference signal.

(A color version of this figure is available in the online journal.)

The absolute calibration for $\mathrm{QUaD}$ is derived by crosscorrelating our CMB temperature maps with those of other experiments and is discussed in more detail in the Data Paper. The calibration values are $0.55 \mathrm{~K} \mathrm{~V}^{-1}$ at $100 \mathrm{GHz}$ and $0.47 \mathrm{~K} \mathrm{~V}^{-1}$ at $150 \mathrm{GHz}$. These values can be estimated based on the measurements of instrument parameters as described in
Section 7. The volts to kelvin calibration factor is given by

$$
s_{\mathrm{K} / \mathrm{V}}=\left(\frac{d Q}{d T} \cdot S_{\mathrm{DC}}\right)^{-1},
$$

where the function $Q(T)$ is the power absorbed by a detector from a beam-filling blackbody source. This in turn is given by

$$
Q(T)=\frac{1}{2} \mathcal{T} \eta_{T} \int A \Omega f_{n}(\nu) B_{\nu}(T) d \nu,
$$

where $\mathcal{T}$ is the atmospheric transmission (Section 7.7), $\eta_{T}$ is the optical efficiency of the telescope optics, $f_{n}(v)$ is the absolute spectral bandpass of the receiver including the optical efficiency of the receiver, $A \Omega=\lambda^{2}$ is the throughput, and $B_{v}(T)$ is the Planck function. The detector responsivity, $S_{\mathrm{DC}}$, in units of $\mathrm{V} \mathrm{W}^{-1}$, is discussed in Section 7.3.

For the $\mathrm{QUaD}$ bands, the derivative $d Q / d T$ evaluated at $T_{\mathrm{CMB}}$ can be calculated to a few percent by evaluating $d B_{v} / d T$ at the band center and approximating the integral as

$$
\left.\frac{d Q}{d T} \approx \frac{1}{2} \mathcal{T} \eta \lambda_{0}^{2} \Delta v \frac{d B_{v}}{d T}\right|_{T=T_{\mathrm{CMB}}, v=\nu_{0}},
$$

where $\eta$ is the total optical efficiency of the system. The telescope efficiency is estimated to be $\sim 0.82$ including loss from the foam cone, the primary mirror, and most significantly shadowing by the secondary mirror. Using average values of the receiver properties at each frequency, this results in the calibration estimates of

$$
\begin{aligned}
s_{\mathrm{K} / \mathrm{V}}(100 \mathrm{GHz})= & 0.51 \frac{\mathrm{K}}{\mathrm{V}} \times\left(\frac{0.94}{\mathcal{T}}\right)\left(\frac{0.82 \cdot 0.26}{\eta_{T} \eta_{R}}\right) \\
& \times\left(\frac{26.5 \mathrm{GHz}}{\Delta v}\right)\left(\frac{3.6 \times 10^{8} \mathrm{~V} / \mathrm{W}}{S_{\mathrm{DC}}}\right) \\
s_{\mathrm{K} / \mathrm{V}}(150 \mathrm{GHz})= & 0.44 \frac{\mathrm{K}}{\mathrm{V}} \times\left(\frac{0.96}{\mathcal{T}}\right)\left(\frac{0.82 \cdot 0.32}{\eta_{T} \eta_{R}}\right) \\
& \times\left(\frac{40.5 \mathrm{GHz}}{\Delta v}\right)\left(\frac{3.1 \times 10^{8} \mathrm{~V} / \mathrm{W}}{S_{\mathrm{DC}}}\right)
\end{aligned}
$$

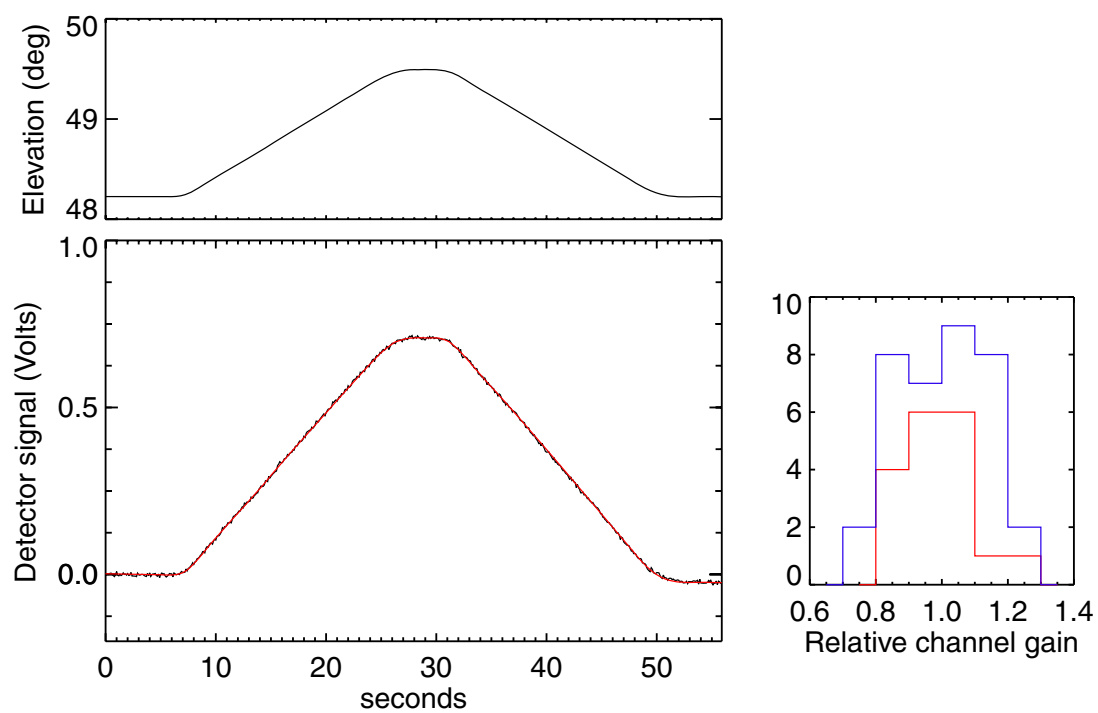

Figure 31. Nodding the telescope in elevation (top) introduces a large signal in the amplified bolometer time stream (bottom) that is used to normalize the relative channel gains. The best-fit model $(1 / \cos (\mathrm{ZA})$ plus a linear drift) is shown in red. The relative channel gains as derived from this el-nod for the $150 \mathrm{GHz}$ (blue) and $100 \mathrm{GHz}$ (red) channels are shown to the right. This distribution results from differences in optical efficiency and detector responsivity.

(A color version of this figure is available in the online journal.) 

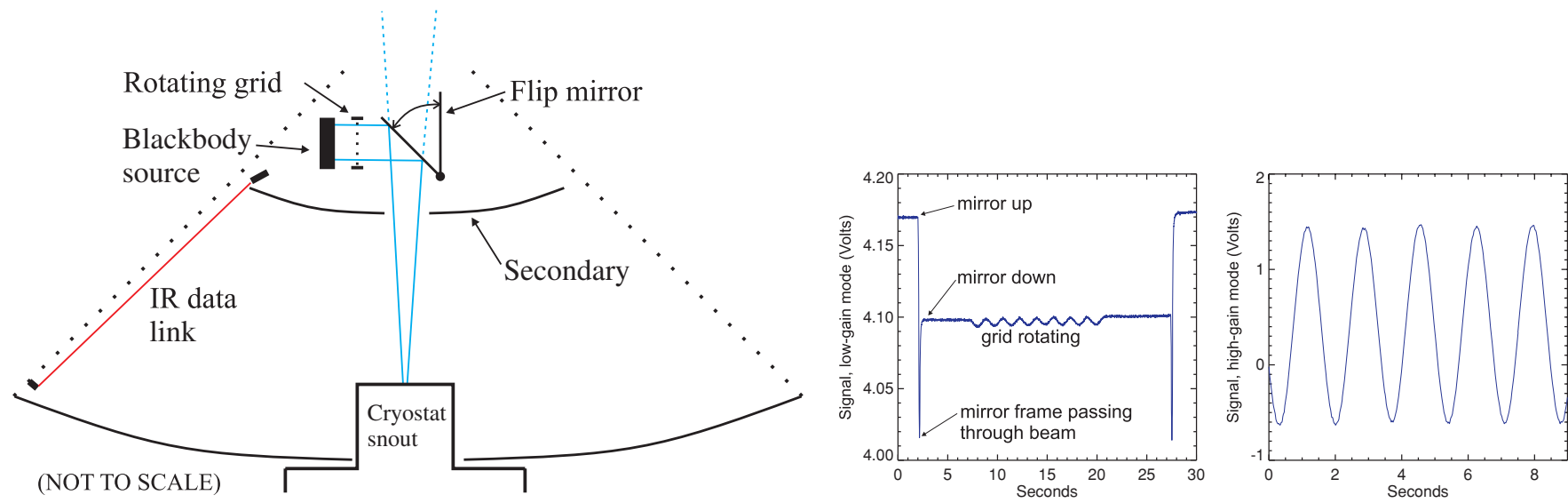

Figure 32. Left: the calibration source is located inside the foam cone, above the secondary mirror. When activated via the infrared link, a flip mirror reflects the signal from a rotating polarizing grid into the telescope beam through the small hole in the secondary. To prevent the calibration signal from saturating the detectors, the flip mirror is actually a small aluminum disk suspended by Kevlar thread within an aluminum frame. Middle: an early test run of the calibration source shows the procedure. Right: a cal source run showing the same channel, taken with the receiver electronics in the usual "high-gain" mode used during observation. The flip mirror area was doubled since the test run to increase the calibration signal.

(A color version of this figure is available in the online journal.)

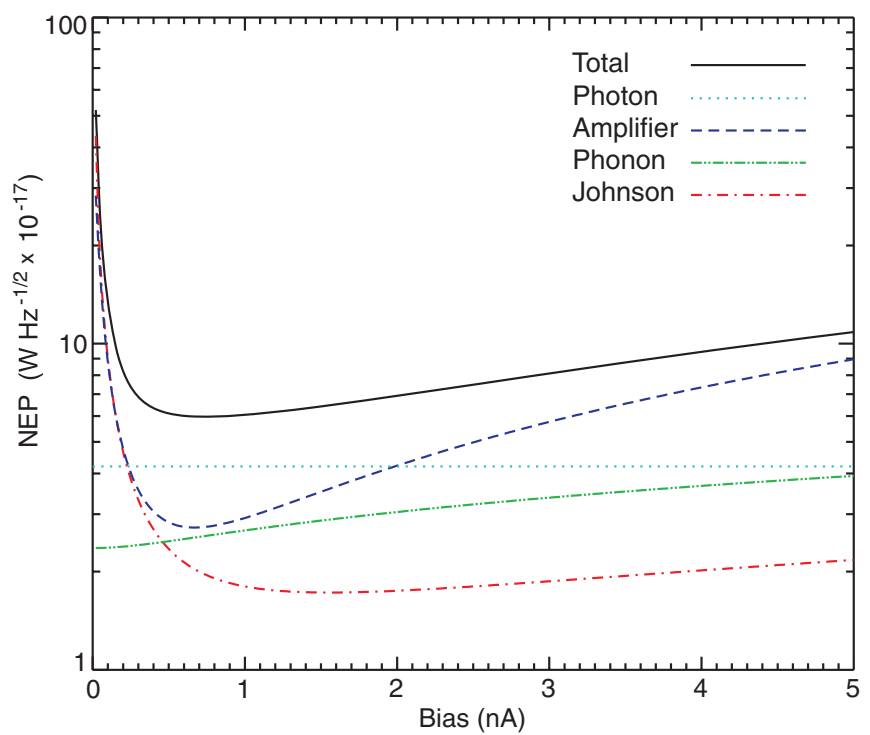

Figure 33. Contributions to the NEP as a function of electrical bias current for a typical $150 \mathrm{GHz}$ detector.

(A color version of this figure is available in the online journal.)

which are in good agreement with the measured calibration values given above.

\section{SENSITIVITY}

The sensitivity of bolometric detectors is limited by Johnson noise from the thermistor and load resistors, phonon noise due to the quantization of the heat conducted between the absorber and the bath, and amplifier noise generated in the readout electronics (Mather 1982, 1984). Additionally there are two sources of noise associated with the quantum nature of the incident optical radiation: shot noise from the discrete photon arrival times and "clumping" noise due to the Boson properties of photons. See, for example, Appendix B of Runyan et al. (2003) for a thorough discussion of this second term.

For millimeter-wave systems, the detector sensitivity is usually quoted as an NEP in $\mathrm{W} \mathrm{Hz}^{-1 / 2}$, computed by dividing the power spectrum in Volts by the detector responsivity (volts/

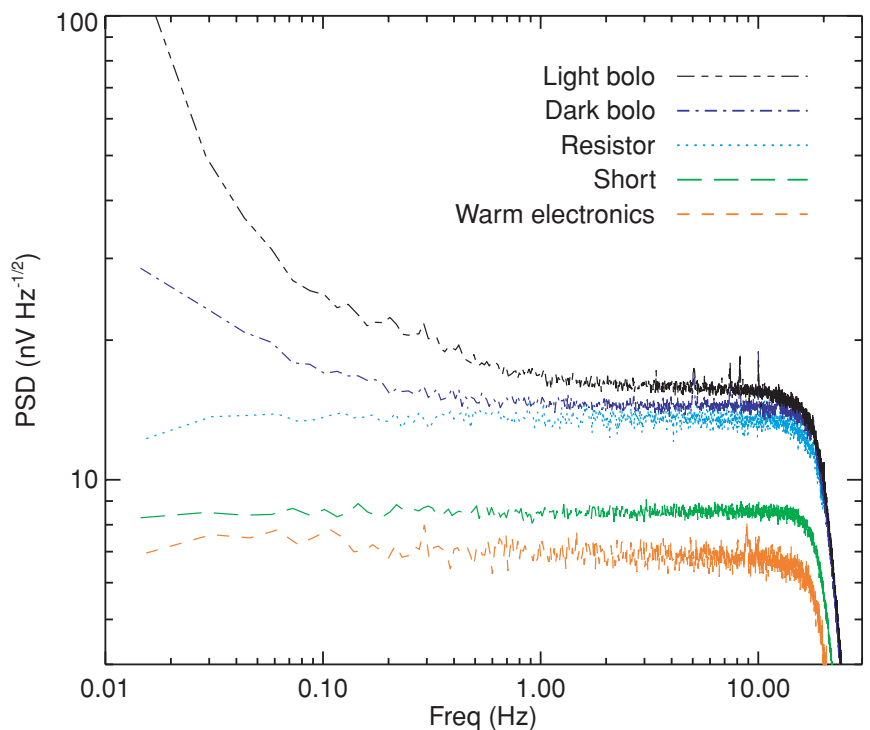

Figure 34. PSD of the noise from various sources. "Warm electronics" accounts for both the readout and the bias generator and was made by connecting a warm $1 \mathrm{k} \Omega$ resistor in place of the cold bolometers and amplifiers. "Short" is a measurement of the noise across a short located on the focal plane and includes contributions from the cold JFETS and the warm amplifiers (but not the bias generator). "Resistor" is a properly biased $10 \mathrm{M} \Omega$ resistor on the focal plane. "Dark bolo" is the readout from a dark bolometer. "Light bolo" is the readout from a $150 \mathrm{GHz}$ bolometer made during normal operation and includes contributions from atmospheric $1 / f$.

(A color version of this figure is available in the online journal.)

watt). Figure 33 shows the expected contributions to the NEP for a typical QUaD detector based on the measured properties of the bolometers and readout electronics. Figure 34 shows the measured noise contributions from the readout electronics.

The sensitivity to CMB temperature fluctuations is quantified as a noise equivalent temperature (NET), which is computed by multiplying the detector voltage noise by the absolute calibration factor in units of kelvin volt ${ }^{-1}$ (Section 9). The NET of a detector is related to the measured NEP as

$$
\mathrm{NET}=\frac{\mathrm{NEP}}{\left.\sqrt{2}(d Q / d T)\right|_{T_{\mathrm{CMB}}}},
$$



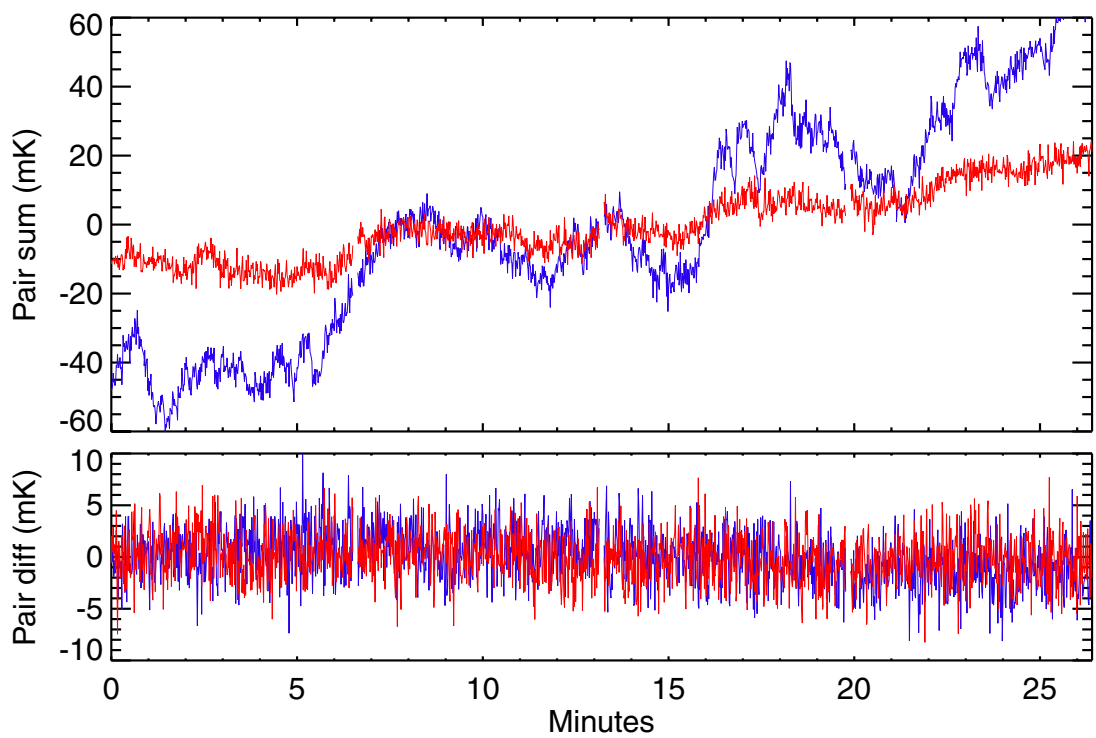

Figure 35. Time-ordered data for a $150 \mathrm{GHz}$ detector pair (blue) and a $100 \mathrm{GHz}$ pair (red) while scanning the telescope during routine CMB observation. Atmospheric $1 / f$ noise, readily apparent in the pair sum (top) is nearly all common mode and cancels upon differencing (bottom).

(A color version of this figure is available in the online journal.)

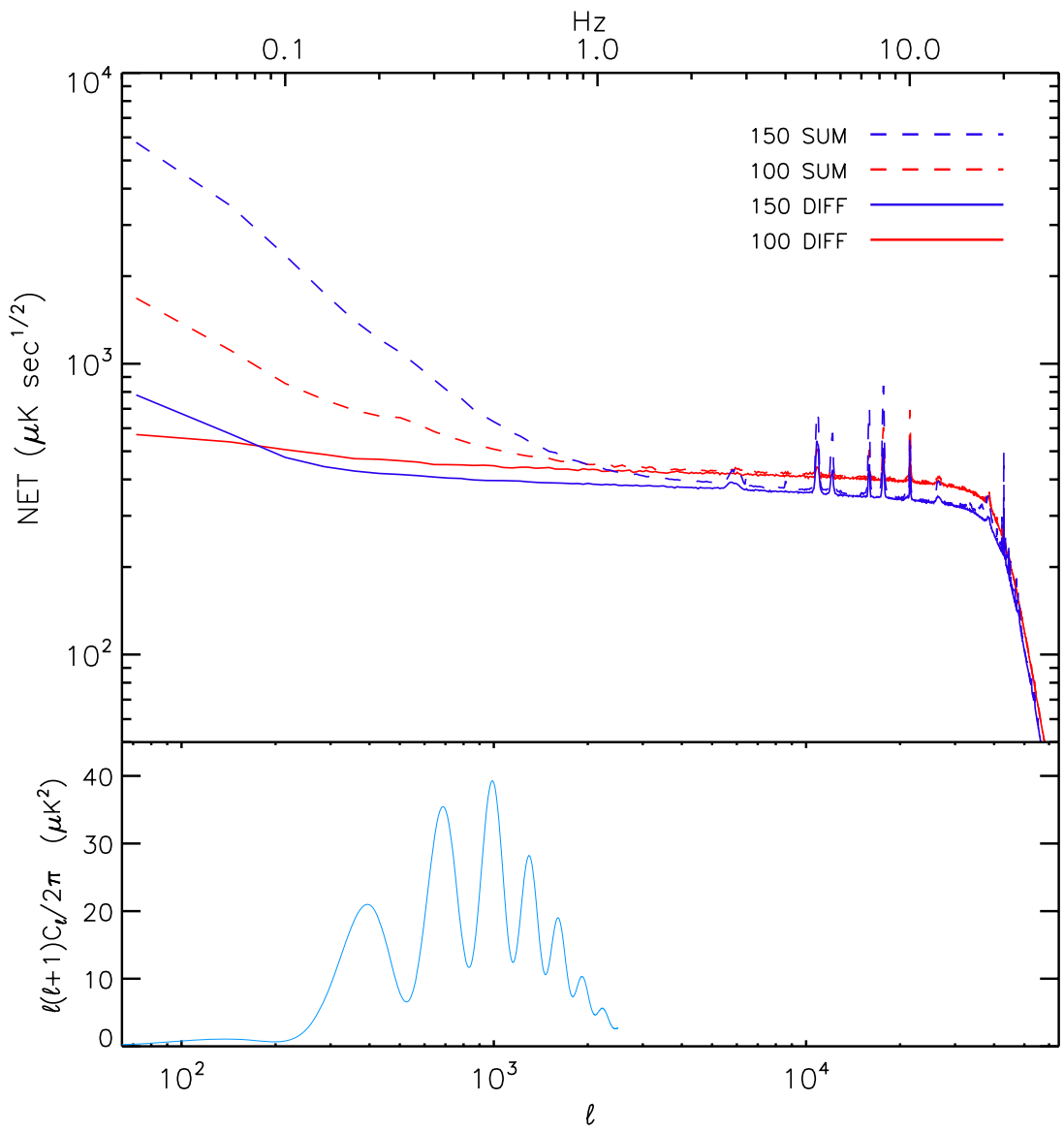

Figure 36. Average per channel NET for PSB sum (dashed) and difference (solid) signals from data taken on 2006 June 23 . The telescope scanned at 0.17 deg s ${ }^{-1}$ on the sky. The E-mode CMB power spectrum is shown below assuming this scanning speed. The spikes seen in the data around approximately $10 \mathrm{~Hz}$ are microphonic resonances. For analysis, the data are low-pass filtered in software. Note that above a frequency of approximately $2.5 \mathrm{~Hz}$, the sensitivity is reduced due to the detector time constants and the antenna beam size (not indicated on this plot).

(A color version of this figure is available in the online journal.)

where the factor of $\sqrt{2}$ in the denominator converts from units of $\mathrm{Hz}^{-1 / 2}$ to $\mathrm{s}^{1 / 2}$ and $d Q / d T$ is defined in Equation (11). This form is frequently used for estimating the sensitivity of a system during the design phase. Similarly, the noise equivalent quanta (NEQ) gives the sensitivity to CMB polarization anisotropies. Since polarization is measured by differencing orthogonal PSB 
pairs, common mode atmospheric noise is greatly reduced (Figure 35). However, the NEQ is degraded relative to the NET by a factor of $(1+\epsilon) /(1-\epsilon)$ (see Equation (5)) which for QUaD is $\sim 1.16$ due to cross-polar leakage.

Figure 36 shows the sensitivity achieved during a typical CMB observing day. The power spectral density (PSD) is estimated by averaging the Fourier transform of each of the approximately 1000 azimuth scans performed during that day. Each scan is approximately $7^{\circ}$ on the sky and takes $40 \mathrm{~s}$. Two PSDs are computed for each PSB pair, one by summing the data, and the other by subtracting. The pair sum and difference PSDs are averaged over all the operational channels in each frequency band. Only minimal filtering is applied to the raw data- a best-fit line is removed from each half scan.

The average NET for a feed horn (PSB pair), not including the effects of atmospheric $1 / f$ noise, is computed from the pair difference data as 440 and $390 \mu \mathrm{K} \sqrt{\mathrm{s}}$ at 100 and $150 \mathrm{GHz}$, respectively over the science band $(0.1-1.0 \mathrm{~Hz})$. The NET for the two bands, including the effects of atmospheric $1 / f$, is computed from the pair sum PSD to be 490 and $580 \mu \mathrm{K} \sqrt{\mathrm{s}}$. Since the atmosphere adds a large amount of $1 / f$-noise, the NET achieved over the course of a season depends on the angular scale of interest, on the filtering used during analysis, and on the weighting used to combine data taken during differing weather conditions. The NEQ for QUaD can be estimated by multiplying the NET computed from the PSB pair difference data by the polarization efficiency factor and is found to be 510 and $450 \mu \mathrm{K} \sqrt{\mathrm{s}}$. Table 3 gives the noise budget and sensitivity for QUaD.

\section{CONCLUSION}

We have presented the design and performance of the QUaD experiment. Stability of key instrumental parameters such as the pointing, beams, and polarization efficiencies have been shown to be at a level that does not interfere with the data collection. Sources of data contamination, including atmospheric noise and sidelobe pickup, are sufficiently well understood to be dealt with effectively - a combination of filtering, field differencing, and data cuts suppresses them to below the level of the instrument sensitivity. QUaD began data collection in early 2005 and completed three seasons of observation before being decommissioned in late 2007. Analysis of these surveys is presented in Ade et al. (2008) and Pryke et al. (2008).

We acknowledge the staff of the Amundsen-Scott South Pole Station and all involved in the United States Antarctic Program for their superb support during the construction and operation of this experiment. Special thanks go to our brave winter-over scientist Robert Schwarz who has spent three consecutive winter seasons with the QUaD. We also acknowledge the tremendous efforts of the Stanford University Physics Department machine shop in the construction of the focal plane assembly. J.R.H. thanks David Chuss for useful comments on this draft and Simon Radford for providing the $350 \mu \mathrm{m}$ tipper data. QUaD is funded by the National Science Foundation in the USA, through grants AST-0096778,ANT-0338138,ANT-0338335, and ANT0338238, by the UK Science and technology Facilities Council (STFC) and its predecessor the Particle Physics and Astronomy
Research Council (PPARC), and by the Science Foundation Ireland.

J.R.H. acknowledges the support of an NSF Graduate Research Fellowship, a Stanford Graduate Fellowship, and a NASA Postdoctoral Fellowship. M.L.B. and A.O. acknowledge the award of PPARC fellowships. P.G.C. is funded by the Fundação para a Ciência e a Tecnologia. S.E.C. acknowledges support from a Stanford Terman Fellowship. J.M.K. acknowledges support from a John B. and Nelly L. Kilroy Foundation Fellowship. C.P. and J.E.C. acknowledge partial support from the Kavli Institute for Cosmological Physics through the grant NSF PHY-0114422. E.Y.W. acknowledges receipt of an NDSEG fellowship. M.Z. acknowledges the support of a NASA Postdoctoral Fellowship. This research was supported in part by appointments to the NASA Postdoctoral Program at the Goddard Space Flight Center (J.R.H.) and the Jet Propulsion Laboratory (M.Z.), administered by Oak Ridge Associated Universities through a contract with NASA.

\section{REFERENCES}

Ade, P. A. R., Pisano, G., Tucker, C., \& Weaver, S. 2006, Proc. SPIE, 6275, (Bellingham, WA: SPIE), 62750U

Ade, P., et al. 2008, ApJ, 674, 22

Bhatia, R. S., et al. 2000, Cryogenics, 40, 685

Birch, J. R., \& Ping, K. F. 1984, Infrared Phys., 24, 309

Bock, J. J. 1994, PhD thesis, AA, California Univ.

Bischoff, C., et al. (CAPMAP Collaboration) 2008, ApJ, 684, 771

Bussmann, R. S., Holzapfel, W. L., \& Kuo, C. L. 2005, ApJ, 622, 1343

Cahill, G., et al. 2004, Proc. SPIE, 5498, 396

Coble, K., et al. 2003, arXiv:astro-ph/0301599

Crill, B. P., et al. 2003, ApJS, 148, 527

Farese, P. C., et al. 2004, ApJ, 610, 625

Gleeson, E., et al. 2005, Infrared Phys. Technol., 46, 493

Glenn, J., et al. 1998, Proc. SPIE, 3357, 326

Hinderks, J. R. 2005, PhD thesis, Stanford University

Holzapfel, W. L., Wilbanks, T. M., Ade, P. A. R., Church, S. E., Fischer, M. L., Mauskopf, P. D., Osgood, D. E., \& Lange, A. E. 1997, ApJ, 479, 17

Jones, W. C., Bhatia, R., Bock, J. J., \& Lange, A. E. 2003, Proc. SPIE, 4855, 227

Kogut, A., et al. 2007, ApJ, 665, 355

Lane, A. P. 1998, BAAS, 30, 883

Lay, O. P., \& Halverson, N. W. 2000, ApJ, 543, 787

Leitch, E. M., Kovac, J. M., Halverson, N. W., Carlstrom, J. E., Pryke, C., \& Smith, M. W. E. 2005, ApJ, 624, 10

Leitch, E. M., et al. 2002a, Nature, 420, 763

Leitch, E. M., et al. 2002b, ApJ, 568, 28

Masi, S., et al. 2006, A\&A, 458, 687

Mather, J. C. 1982, Appl. Opt., 21, 1125

Mather, J. C. 1984, Appl. Opt., 23, 584

Montroy, T. E., et al. 2006, ApJ, 647, 813

Murphy, J. A., et al. 2005, Int. J. Infrared Mm Waves, 26, 505

O’Sullivan, C., et al. 2008, Infrared Phys. Technol., 51, 277

Page, L., et al. 2007, ApJS, 170, 335

Peterson, J. B., Radford, S. J. E., Ade, P. A. R., Chamberlin, R. A., O'Kelly, M. J., Peterson, K. M., \& Schartman, E. 2003, PASP, 115, 383

Pryke, C., et al. 2009, ApJ, 692, 1247

Readhead, A. C. S., et al. 2004, Science, 306, 836

Runyan, M. C. 2002, PhD thesis, California Institute of Technology

Runyan, M. C., et al. 2003, ApJS, 149, 265

Sudiwala, R. V., Griffin, M. J., \& Woodcraft, A. L. 2002, Int. J. Infrared Mm Waves, 23, 545

Woodcraft, A. L., et al. 2003, Appl. Opt., 42, 5009

Wu, J. H. P., et al. 2007, ApJ, 665, 55

Yoon, K. W., et al. 2006, Proc. SPIE, 6275, 62751K

Zaldarriaga, M., \& Seljak, U. 1997, Phys. Rev. D, 55, 1830

Zemcov, M. 2006, PhD thesis, Cardiff University 